\title{
A Multiphysics Approach for Modeling Early Atherosclerosis
}

\author{
M. P. Thon • A. Hemmler • A. Glinzer • M. Mayr • M. Wildgruber • \\ A. Zernecke-Madsen • M. W. Gee
}

Received: date / Accepted: date

\begin{abstract}
This work is devoted to the development of a mathematical model of the early stages of atherosclerosis incorporating processes of all time scales of the disease and to show their interactions. The cardiovascular mechanics is modeled by a fluid-structure interaction approach coupling a non-Newtonian fluid to a hyperelastic solid undergoing anisotropic growth and a change of its constitutive equation. Additionally, the transport of low-density lipoproteins and its penetration through the endothelium is considered by a coupled set of advectiondiffusion-reaction equations. Thereby, the permeability of the endothelium is wall-shear stress modulated resulting in a locally varying accumulation of foam cells triggering a novel growth and remodeling formulation.
\end{abstract}

M. P. Thon · A. Hemmler · M. Mayr · M. W. Gee (四) Mechanics \& High Performance Computing Group, Technical University of Munich, Parkring 35, 85748 Garching b. München, Germany

E-mail: gee@tum.de

A. Glinzer · M. Wildgruber

Department of Radiology, Technical University of Munich, Ismaningerstraße 22, 81675 München, Germany

\section{Wildgruber}

Translational Research Imaging Center, Westfälische Wilhelms Universität Münster, Albert-Schweitzer-Campus 1, 48149 Münster, Germany

\section{A. Zernecke-Madsen}

Institute of Experimental Biomedicine, University Hospital Würzburg, Josef-Schneider-Straße 2, 97080 Würzburg, Germany

Present Address:

M. Mayr

Sandia National Laboratories, 7011 East Avenue, MS 9158, Livermore, CA 94550, USA
The model is calibrated and applied to an murinespecific case study and a qualitative validation of the computational results is performed. The model is utilized to further investigate the influence of the pulsatile blood flow and the compliance of the artery wall to the atherosclerotic process. The computational results imply that the pulsatile blood flow is crucial, whereas the compliance of the aorta has only a minor influence on atherosclerosis. Further, it is shown that the novel model is capable to produce a narrowing of the vessel lumen inducing an adaption of the endothelial permeability pattern.

Keywords atherosclerosis - multiphysics $\cdot$ fluidstructure interaction - growth and remodeling . cardiovascular mechanics $\cdot$ hemodynamics $\cdot$ pulsatile flow

\section{Introduction}

Atherosclerosis is an inflammatory disease resulting in the pathological alteration of the intima and media of arteries such as e.g. the aorta. Inducing sequelae like stroke, heart attack or angina, atherosclerosis is the leading cause of death in western societies. It is characterized by an accumulation of inflammatory cells and lipids in the intima and media leading to their thickening and hence to a narrowing of the vessel lumen. It is now wellaccepted that a significant first step for the initiation of the early atherosclerotic process is a dysfunction of the endothelium allowing the penetration of low-density lipoproteins (LDL) through the monolayer of endothelial cells into the vessel wall. Thereby, the role of the endothelium is crucial since it acts as a transportation barrier between the lumen and the intima. LDL in the vessel wall is prone to oxidative modifications initiating 
the inflammatory processes. The inflammation further triggers a complex biochemical immune response leading to the migration of monocytes into the artery wall inducing their differentiation into macrophages. Macrophages ingest the modified LDL and can transform to so-called foam cells. These lipid-laden foam cells accumulate resulting in the development of atherosclerotic plaques and hence a thickening of the artery wall. A significant narrowing of the vessel lumen can occur and a potential rupture of the plaque can lead to subsequent diseases like stroke or myocardial infarction $[84,92,28,10]$.

Over the recent decades more and more evidence was found that low wall-shear stresses (WSS), resulting from flow recirculations and oscillatory flows, locally trigger atherosclerosis by an increased permeability of the endothelium with respect to LDL. However, the concrete interplay of hemodynamic forces, endothelial permeability and atherosclerosis progression is not yet fully understood $[80,82]$.

To study the influence of the mechanobiology to atherosclerosis a broad spectrum of mathematical and computational models were established. For a general overview of existing models and their specific applications, see e.g. [79,45,101] and therein. A great challenge for computational models are different time scales involved in the atherosclerotic process. On the one hand the LDL penetration, the inflammatory processes as well as the foam cell accumulation is on the time scale of weeks, months or years. On the other hand the hemodynamics and therefore the long-time endothelial permeability is governed by the time scale of cardiac cycles being in the range of seconds.

Long time scale models can roughly be divided into the ones focusing on LDL penetration and the ones emphasizing the biochemical reactions of the inflammatory processes. Many of the LDL penetration models consider the transmural flow driven by the pressure gradient across the endothelium as well as the pressure driven transportation within the vessel wall to be of importance, see e.g. $[81,96,106]$. In contrast, others model the penetration and transportation as a purely diffusive process [46,11]. A broad spectrum of models for the inflammatory processes exist $[13,34,32,78,17,11]$. Some consider only a few key species [13,11], where others identify up to 16 important players [34]. Some identified chemotaxis to be crucial $[13,34]$, where others restrict themselves to pure ordinary differential equation models [78]. Since from a medical point of view the complex biochemical processes involved are not yet fully understood and since a quantitative validation of the mathematical models by experiments is not yet achieved, it is difficult to distinguish the validity of these models. Additionally, some of the models also consider the plaque develop- ment process $[11,34,96]$ in terms of heuristic growth laws. Only very few models consider all of the beforehand mentioned processes at the large time scale. One can highlight the work in [11] considering the transmural flow within the artery wall, a simple but convenient biochemical reaction model and an induced heuristic growth.

Small time scale models in contrast focus on a physiological description of the cardiovascular mechanics. This includes mainly two aspect missing in most of the large time scale models: pulsatile blood flow and compliance of the vessel wall. It is frequently stated that pulsatile flow should not be neglected $[58,66,93]$ lying in contradiction to many models, which commonly assume stationary blood flows. The influence of the compliance of the artery is usually investigated using fluid-structure interaction (FSI) models allowing for a physiologically more realistic deformation of the artery wall $[19,58,71$, $107,31]$. However, the influence of the compliance on the atherosclerotic process has not been considered much [21]. For the small time scales one can highlight the work in [58], were a non-stationary FSI simulation, a model of the species transportation and penetration as well as a linked model of the transmural flow is considered. The back coupling from the large time scale, i.e. the plaque development process and subsequent geometry changes crucial to atherogenesis are not included therein.

A suitable multiscale in time strategy is necessary to bring together the aforementioned small and large time scale phenomena. General multiscale frameworks exist, see e.g. [31], but a suitable framework for atherosclerosis is not yet established. As a first step [58,93] considered the influence of the flow pattern to LDL penetration, but not vice versa. In contrast, [96,11] modeled the large time scale growth process and studied the induced changes to hemodynamics, both assuming stationary flows and phenomenological growth laws. Still, [96] shows the back-coupling from the large time scale due to growth being of major importance. It may explain that the so-called fatty streak formation observable in early stages of the disease is a result of the adjusted LDL penetration due to the thickened artery wall altering the blood flow.

In this contribution, the objective is to develop a mathematical model of early atherosclerosis incorporating all beforehand mentioned phenomena and include and discuss their interactions. Therefore, a multiphysics model of the cardiovascular mechanics as well as for the transportation and penetration of LDL is developed focusing on the following aspects: pulsatile blood flow, compliant artery wall, WSS dependent migration of LDL and growth and remodeling. To achieve meaningful results the model is calibrated to and solved for 
a murine-specific geometry segmented from an in vivo magnetic resonance angiography. The proposed model is designed for the small time scale and is capable to physiologically adapt to the long time processes of plaque development and the induced narrowing of the blood vessel. Therefore a state of the art FSI model of the cardiovascular mechanics as well as a sequentially coupled scalar transport model including a novel way for calibrating the law for LDL penetration is established. A simple phenomenological model of the inflammatory processes is utilized to represent the large time scale processes of foam cell accumulation triggering a novel growth and remodeling formulation. The model is able to reproduce important cardiovascular quantities gained by measurements and simulations of previous studies. We further utilize the model to study the interaction between the two time scales. In particular, the question of the influence of pulsatile blood flow and vessel compliance on atherosclerosis are addressed.

The paper is organized as follows. In the next Section we give an overview of the simplified model of the atherosclerotic process and its mathematical formulation describing the cardiovascular mechanics, species migration as well as the growth and remodeling processes. Section 3 briefly presents the numerical procedure. In section 4 the model is calibrated to a murine-specific case and computational results are presented. Finally, results are discussed in section 5 and critically reflected in sections 6 and 7 .

\section{Modeling}

\subsection{Overview of the simplified model}

To reproduce the atherosclerotic process in a mathematical model reasonable simplifications and assumptions have to be made. Here the main interest is to study the mechanobiological influence of the cardiovascular mechanics driven by the hemodynamics on the atherosclerotic process and vice versa. Therefore, we consider the following assumptions:

- The hemodynamics is governed by the pulsatile blood flow interacting with the elastic artery wall.

- LDL molecules are transport by advection and diffusion in the lumen and solely by diffusion in the artery wall.

- The initiator of the atherosclerotic inflammation is the migration of LDL through the endothelium into the artery wall.

- The endothelium has an increased permeability with respect to LDL at regions of low wall shear stresses.
- In the artery wall LDL triggers a series of bio-chemical processes which lead to the production of foam cells.

- The accumulation of foam cells in the artery wall leads to a thickening of the artery wall with an induced change of its mechanical properties.

- The thickening of the artery wall is considered to be stress free in the reference configuration.

For a schematic overview of the simplified model and the considered main aspects in the atherosclerotic process, see Figure 1.

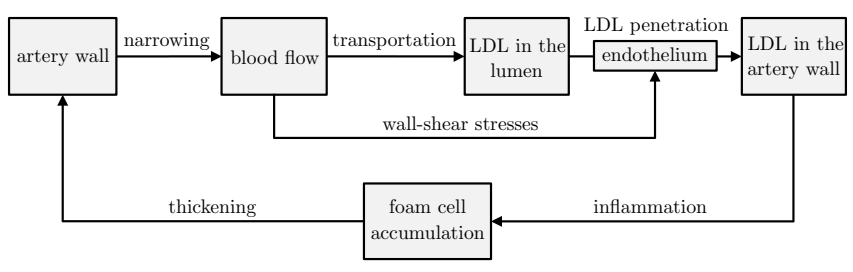

Fig. 1 Schematic overview of the simplified model and the considered main aspects in the atherosclerotic process.

The simplified model of atherosclerosis is represented by a mathematical formulation as follows. The governing equations are a coupled fluid-structure-advectiondiffusion-reaction model, that we subsequently denote as fluid-structure-scalar-scalar interaction (FS3I). It can be subdivided into a model of the interaction of the blood flow with the artery wall and a model of the transport and reactions of the key species involved. The former is realized by a FSI approach coupling an incompressible non-Newtonian fluid including embedded three-element Windkessels with a hyperelastic structure, which undergoes species concentration dependent anisotropic growth and a species concentration dependent remodeling of its constitutive equation. The transportation of LDL with the blood flow is governed by the advection-diffusion equation and its migration through the endothelium into the artery wall by a WSS dependent, modified version of the Kedem-Katchalsky equations. LDL in the artery wall is modeled by the diffusion-reaction equation leading to the production of the growth inducing species of foam cells.

\subsection{Notations and domain overview}

In the following domains are denoted by $\Omega \subset \mathbb{R}^{3}$ and boundaries are denoted by $\Gamma \subset \partial \Omega$. They undergo finite deformations in time $t$, which is explicitly expressed by $\Omega(t)$ and $\Gamma(t)$, respectively, when of particular interest. The variables for space are $\mathbf{X}$ and $\mathbf{x}$, which denote the material and spatial coordinates, respectively, where the 
bolding denotes a vector or tensor valued quantity. The displacements and velocities of a material point $\mathbf{X}$ at time $t$ are denoted by $\mathbf{d}(t, \mathbf{X})$ and $\mathbf{u}(t, \mathbf{X})=\frac{\mathrm{d}}{\mathrm{d} t} \mathbf{d}(t, \mathbf{X})$, respectively.

To account for the deformable domain of the fluid within the FSI problem an Arbitrary-Lagrangian-Eulerian (ALE) observer is utilized. The ALE domain is thereby denoted by $\Omega_{0}^{\mathcal{G}}$ since it is convenient to think of it as the domain of the grid $\mathcal{G}$ of the ALE fluid. When reformulating an Eulerian problem describing the motion of a quantity $(\star)$ to an ALE observer, the so-called ALE time derivative $\left.\frac{\partial}{\partial t}(\star)\right|_{\chi}$ has to be exploited [26,27]. We usually omit the time and space dependencies to ease notation, except in cases where it is crucial.

In the context of atherosclerosis a special focus lies on bifurcations of large arteries where atherosclerotic plaques are frequently located. Hence, in this context the aortic arch with the branching subclavian and common carotid arteries is utilized as the computational region of interest $\Omega$. The overall computational domain $\Omega$ can be subdivided into the domain of the lumen and the domain of the artery wall. Within the lumen the computational domains of the fluid $\Omega^{\mathcal{F}}$, of the ALE observer $\Omega^{\mathcal{G}}$ and of the scalar-valued concentration in the fluid (fluidscalar) $\Omega^{\mathcal{F S}}$ are located. Within the artery wall the domains of the structure $\Omega^{\mathcal{S}}$ and of the scalar-valued concentrations in the structure (structure-scalars) $\Omega^{\mathcal{S}}$ are situated, see Figure 2.

To easily distinguish the affiliation of a quantity $(\star)$ to the five computational domains its name is placed as a superscript, i.e. the quantity is denoted by $(\star)^{\mathcal{F}}$, $(\star)^{\mathcal{S}},(\star)^{\mathcal{G}},(\star)^{\mathcal{F} \mathcal{S}}$ or $(\star)^{\mathcal{S S}}$. Names of quantities are indicated as subscript. Each computational domains contains a boundary $\Gamma_{\text {In }}$ and $n_{\text {Out }} \geq 1$ boundaries $\Gamma_{\text {Out }, i}$, $i=1, \ldots, n_{\text {Out }}$. The fluid-structure interface as well as the (fluid-scalar)-(structure-scalar) interface, short the fluid-structure-scalar-scalar interface corresponding to the endothelium is denoted by $\Gamma_{\mathrm{FS} 3 \mathrm{I}}$. The boundary connecting the outer artery wall with the surrounding tissue is called $\Gamma_{\text {Wall }}$. For a schematic overview of the different domains and boundaries, see Figure 2.

\subsection{Cardiovascular mechanics}

The cardiovascular mechanics is modeled by a FSI method [19,70,61,107,57] coupling an incompressible non-Newtonian fluid including embedded three-element Windkessels with a hyperelastic solid undergoing finite deformations, anisotropic growth and a change of its constitutive equation.

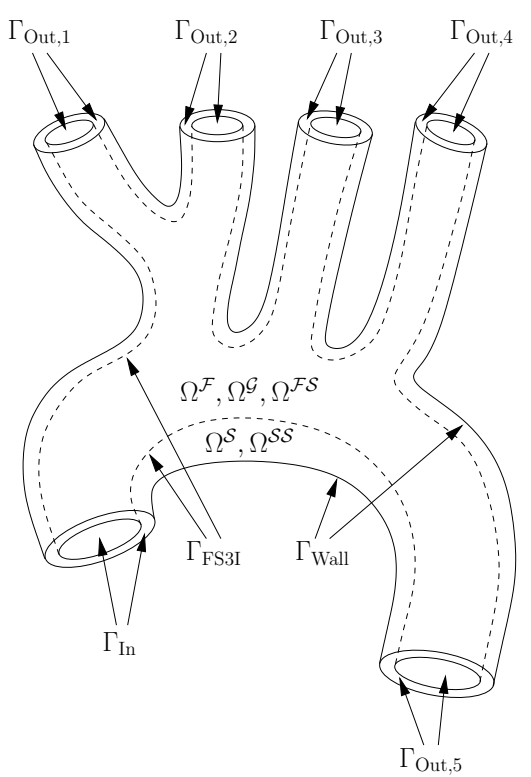

Fig. 2 Schematic overview of the domains and boundaries of an aortic arch: fluid domain $\Omega^{\mathcal{F}}$, structure domain $\Omega^{\mathcal{S}}$, ALE observer domain $\Omega^{\mathcal{G}}$, fluid-scalar domain $\Omega^{\mathcal{F} \mathcal{S}}$, structurescalars domain $\Omega^{\mathcal{S S}}$, inlet boundary $\Gamma_{\mathrm{In}}$, outlet boundaries $\Gamma_{\text {Out }, i}(i=1, \ldots, 5)$, fluid-structure-scalar-scalar interaction interface $\Gamma_{\mathrm{FS} 3 \mathrm{I}}$ and outer wall boundary $\Gamma_{\mathrm{Wall}}$.

\subsubsection{Fluid model of the blood}

We model blood as an incompressible non-Newtonian fluid. The blood flow on the deformable domain $\Omega^{\mathcal{F}}(t)$ is governed by the incompressible Navier-Stokes equations in an ALE frame

$$
\begin{aligned}
\left.\varrho^{\mathcal{F}} \frac{\partial}{\partial t} \mathbf{u}^{\mathcal{F}}\right|_{\chi} & +\varrho^{\mathcal{F}}\left(\left(\mathbf{u}^{\mathcal{F}}-\mathbf{u}^{\mathcal{G}}\right) \cdot \nabla\right) \mathbf{u}^{\mathcal{F}} \\
& -2 \eta^{\mathcal{F}}\left(\mathbf{u}^{\mathcal{F}}\right) \nabla \cdot \varepsilon\left(\mathbf{u}^{\mathcal{F}}\right)+\nabla p^{\mathcal{F}}=\mathbf{0}
\end{aligned}
$$

$\nabla \cdot \mathbf{u}^{\mathcal{F}}=0$

where $\left.\frac{\partial}{\partial t} \mathbf{u}^{\mathcal{F}}\right|_{\boldsymbol{\chi}}$ denotes the ALE time derivative of the fluid velocities $\mathbf{u}^{\mathcal{F}}$, see $[26,27]$. The motion of the ALE observer is described in section 2.3.4 and its velocity field is denoted by $\mathbf{u}^{\mathcal{G}}$. The constant $\varrho^{\mathcal{F}}$ and $\varepsilon\left(\mathbf{u}^{\mathcal{F}}\right)=$ $\frac{1}{2}\left(\nabla \mathbf{u}^{\mathcal{F}}+\left(\nabla \mathbf{u}^{\mathcal{F}}\right)^{T}\right)$ are the mass density and strain rate tensor, respectively. Blood exhibits a shear-thinning property, i.e. a decrease of its viscosity when its strainrate increases $[16,66,14]$. We use the Carreau-Yasuda model to account for the shear-thinning property of blood $[16,9,2,37]$

$\eta^{\mathcal{F}}\left(\mathbf{u}^{\mathcal{F}}\right)=\eta_{\infty}+\frac{\eta_{0}-\eta_{\infty}}{\left(1+\left(\kappa \dot{\gamma}\left(\mathbf{u}^{\mathcal{F}}\right)\right)^{b}\right)^{a}}$,

where $\eta_{\infty}, \eta_{0}, \kappa, a$ and $b$ are constants and $\dot{\gamma}\left(\mathbf{u}^{\mathcal{F}}\right)=$ $\sqrt{2 \operatorname{tr}\left(\varepsilon\left(\mathbf{u}^{\mathcal{F}}\right)^{2}\right)}$ is the shear-rate of the fluid. The CarreauYasuda model is recommended for low and mid-range 
velocities [51] and is especially well-suited for our application.

On $\Gamma_{\text {In }}^{\mathcal{F}}$ the following Dirichlet condition is applied

$\mathbf{u}^{\mathcal{F}}(t, \mathbf{x})=-Q_{\mathrm{In}}^{\mathcal{F}}(t) g(t, \mathbf{x}) \mathbf{n}_{\text {In }}^{\mathcal{F}}$,

where in general $\mathbf{n}_{\left(\star_{2}\right)}^{\left(\star_{1}\right)}$ denotes the outside pointing unit surface normal on the deformed surface $\Gamma_{\left(\star_{2}\right)}^{\left(\star_{1}\right)}$. The scalar-valued function $g(t, \mathbf{x})$ corresponds to the applied velocity profile and $Q_{\text {In }}^{\mathcal{F}}(t)$ to the total volume influx. Thereby $Q_{\mathrm{In}}^{\mathcal{F}}(t)$ is a $T_{\mathrm{Cycl}}$-periodical function, to regard the pulsatile nature of blood flow with a cardiac cycle duration of $T_{\mathrm{Cycl}}$. We want to account for the Windkessel effect of succeeding arteries to achieve a physiological pressure range for the fluid. Therefore, time varying pressures $p_{\mathrm{WK}, i}^{\mathcal{F}}$ from the underlying Windkessel models (see section 2.3.2) are applied as tractions on each of the outflow boundaries $\Gamma_{\text {Out }, i}^{\mathcal{F}}$ :

$\boldsymbol{\sigma}^{\mathcal{F}} \mathbf{n}_{\text {Out }, i}^{\mathcal{F}}=-p_{\mathrm{WK}, i}^{\mathcal{F}} \mathbf{n}_{\text {Out }, i}^{\mathcal{F}}$,

where $\boldsymbol{\sigma}^{\mathcal{F}}=-p^{\mathcal{F}} \mathbf{1}+2 \eta^{\mathcal{F}}\left(\mathbf{u}^{\mathcal{F}}\right) \boldsymbol{\varepsilon}\left(\mathbf{u}^{\mathcal{F}}\right)$ is the Cauchy stress tensor of the fluid.

\subsubsection{Windkessel model of the blood pressure}

To achieve a physiological pressure range of the fluid and to physiologically split the total flux to the different bifurcations, a separate three-element Windkessel model $[102,77,105,50]$ is used on each of the outflow boundaries $\Gamma_{\text {Out }, i}^{\mathcal{F}}$ :

$$
\begin{aligned}
& C_{i} \frac{\mathrm{d}}{\mathrm{d} t} p_{\mathrm{WK}, i}^{\mathcal{F}}(t)+\frac{1}{R_{\mathrm{P}, i}} p_{\mathrm{WK}, i}^{\mathcal{F}}(t) \\
& \quad=C_{i} R_{\mathrm{C}, i} \frac{\mathrm{d}}{\mathrm{d} t} Q_{\mathrm{Out}, i}^{\mathcal{F}}(t)+\left(1+\frac{R_{\mathrm{C}, i}}{R_{\mathrm{P}, i}}\right) Q_{\mathrm{Out}, i}^{\mathcal{F}}(t),
\end{aligned}
$$

where $Q_{\text {Out }, i}^{\mathcal{F}}(t)=\int_{\Gamma_{\text {Out }, i}^{\mathcal{F}}} \mathbf{u}^{\mathcal{F}}(t) \cdot \mathbf{n}_{\text {Out }, i}^{\mathcal{F}} \mathrm{d} a$ is the current outflux through $\Gamma_{\text {Out }, i}^{\mathcal{F}}$. The constants $R_{\mathrm{C}, i}, R_{\mathrm{P}, i}$ and $C_{i}$ correspond to the characteristic resistance, peripheral resistance and artery compliance of the successive artery network, respectively. They have to be fitted to the specific case to produce physiologically meaningful results, see section 4.1 .

\subsubsection{Structure model of the artery wall}

The artery wall is a multi-component structure that also contains a fluid phase [106]. Here, its mechanical response is modeled through a anisotropic hyperelastic material law [47], while we allow for movement of species inside the artery tissue, see Section 2.4.2. Hence, we follow the frequently used approach of modeling the artery wall as a solid $[19,71,58,21,31]$ governed by the balance of linear momentum on $\Omega_{0}^{\mathcal{S}}$

$\varrho_{0}^{\mathcal{S}} \frac{\mathrm{d}^{2}}{\mathrm{~d} t^{2}} \mathbf{d}^{\mathcal{S}}-\nabla \cdot\left(\mathbf{F}^{\mathcal{S}} \mathbf{S}^{\mathcal{S}}\left(\mathbf{C}^{\mathcal{S}}\right)\right)=\mathbf{0}$

with $\mathbf{F}^{\mathcal{S}}=\mathbf{1}+\boldsymbol{\nabla} \mathbf{d}^{\mathcal{S}}$ being the deformation gradient, $\mathbf{C}^{\mathcal{S}}=\left(\mathbf{F}^{\mathcal{S}}\right)^{T} \mathbf{F}^{\mathcal{S}}$ the right Cauchy-Green deformation tensor and $\mathbf{S}^{\mathcal{S}}$ the second Piola-Kirchhoff stress tensor. The constant $\varrho_{0}^{\mathcal{S}}$ is the reference mass density of the artery wall.

To incorporate the effect of the tissue surrounding the aorta, a spring and dashpot combination on $\Gamma_{\text {Wall }}^{\mathcal{S}}$ $[71,67]$ is applied:

$\left(\mathbf{F}^{\mathcal{S}} \mathbf{S}^{\mathcal{S}}\right) \mathbf{N}_{\text {Wall }}^{\mathcal{S}}=-k_{\text {Wall }}^{\mathcal{S}} \mathbf{d}^{\mathcal{S}}-c_{\text {Wall }}^{\mathcal{S}} \mathbf{u}^{\mathcal{S}}$

where in general $\mathbf{N}_{\left(\star_{2}\right)}^{\left(\star_{1}\right)}$ denotes the outside pointing unit surface normal on the undeformed surface $\Gamma_{\left(\star_{2}\right)}^{\left(\star_{1}\right)}$. The constants $k_{\text {Wall }}^{\mathcal{S}}$ and $c_{\text {Wall }}^{\mathcal{S}}$ are the spring stiffness and dashpot viscosity of the surrounding tissue, respectively. To respect the influence of the succeeding aortic tissue on all boundaries $\Gamma_{\mathrm{Out}, i}^{\mathcal{S}}$ sliding springs and dashpots acting only in the direction of the surface normal and allowing a free movement in the boundary plane are applied:

$$
\begin{aligned}
\left(\mathbf{F}^{\mathcal{S}}\right. & \left.\mathbf{S}^{\mathcal{S}}\right) \mathbf{N}_{\text {Out }, i}^{\mathcal{S}} \\
& =\mathbf{N}_{\text {Out }, i}^{\mathcal{S}} \cdot\left(-k_{\text {Out }}^{\mathcal{S}} \mathbf{d}^{\mathcal{S}}-c_{\text {Out }}^{\mathcal{S}} \mathbf{u}^{\mathcal{S}}\right) \mathbf{N}_{\text {Out }, i}^{\mathcal{S}}
\end{aligned}
$$

where $k_{\text {Out }}^{\mathcal{S}}$ and $c_{\text {Out }}^{\mathcal{S}}$ are the spring stiffness and dashpot viscosity of the succeeding aortic tissue, respectively. On the boundary $\Gamma_{\text {In }}^{\mathcal{S}}$, a zero displacement Dirichlet condition is applied.

\section{Growth}

In addition to the elastodynamics we consider the nonelastic process of growth due to the deposition of foam cells in the atherogenesis [101]. We assume the growth of the artery wall to be stress free in the reference configuration [87] and hence utilize a multiplicative split of the deformation gradient $\mathbf{F}^{\mathcal{S}}$ of the structure into an elastic part $\mathbf{F}_{\text {Elast }}^{\mathcal{S}}$ and a growth part $\mathbf{F}_{\text {Growth }}^{\mathcal{S}}[60,1]$ :

$$
\mathbf{F}^{\mathcal{S}}=\mathbf{F}_{\text {Elast }}^{\mathcal{S}} \mathbf{F}_{\text {Growth }}^{\mathcal{S}}
$$

The so introduced growth configuration is denoted by $\Omega_{\text {Growth }}^{\mathcal{S}}(t)$ and the respective coordinates are denoted by $\hat{\chi}$. To respect the stress-free nature of the growth, the second Piola-Kirchhoff stress tensor $\mathbf{S}^{\mathcal{S}}$ in equation (7) is computed as a pull-back of the elastic stresses $\mathbf{S}_{\text {Elast }}^{\mathcal{S}}$, i.e. $\mathbf{S}^{\mathcal{S}}=\left(\mathbf{F}_{\text {Growth }}^{\mathcal{S}}\right)^{-1} \mathbf{S}_{\text {Elast }}^{\mathcal{S}}\left(\mathbf{F}_{\text {Growth }}^{\mathcal{S}}\right)^{-T}$. We further 
assume the artery wall to be hyperelastic with strainenergy density function $\Psi^{\mathcal{S}}$, i.e. the elastic stresses can be calculated by

$$
\begin{aligned}
& \mathbf{S}_{\text {Elast }}^{\mathcal{S}}=2 \frac{\partial}{\partial \mathbf{C}_{\text {Elast }}^{\mathcal{S}}} \Psi^{\mathcal{S}}\left(\mathbf{C}_{\text {Elast }}^{\mathcal{S}}\right), \\
& \mathbf{C}_{\text {Elast }}^{\mathcal{S}}=\left(\mathbf{F}_{\text {Elast }}^{\mathcal{S}}\right)^{T} \mathbf{F}_{\text {Elast }}^{\mathcal{S}} .
\end{aligned}
$$

The natural direction of growth is the luminal direction, as it is induced by the accumulation of foam cells in the intima and the adjacent media. Furthermore, growth of the aorta in the axial or circumferential direction would stretch collagen and elastin fibers inside the artery wall and hence introduce additional wall stresses. For a better understanding of the theory of anisotropic growth we first assume the unit radial direction ra, the unit axial direction ax and the unit circumferential direction ci to be constants (an assumption that we drop in the subsequent discussion). Hence, we postulate the following form of the growth deformation gradient [56]:

$\mathbf{F}_{\text {Growth }}^{\mathcal{S}}=\vartheta\left(c_{\mathrm{FC}}^{\mathcal{S}}\right) \mathbf{r a} \otimes \mathbf{r a}+\mathbf{a x} \otimes \mathbf{a x}+\mathbf{c i} \otimes \mathbf{c i}$

where the scalar-valued function $\vartheta\left(c_{\mathrm{FC}}^{\mathcal{S}}\right)$ is the growth factor and does depend on the local (mass) concentration of the growth inducing species $c_{\mathrm{FC}}^{\mathcal{S}}$. Since the set $\{\mathbf{r a}, \mathbf{a x}, \mathbf{c i}\}$ is an orthonormal basis of $\mathbb{R}^{3}$ we can simplify equation (12) to

$\mathbf{F}_{\text {Growth }}^{\mathcal{S}}=\mathbf{1}+\left(\vartheta\left(c_{\mathrm{FC}}^{\mathcal{S}}\right)-1\right) \mathbf{r a} \otimes \mathbf{r a}$,

which now only depends on the unit radial direction ra. The model of the foam cells is described in section 2.4.2. For the computation of the growth factor $\vartheta\left(c_{\mathrm{FC}}^{\mathcal{S}}\right)$ we exploit the idea that the increase of volume $\Delta V_{\text {Growth }}(t)$ due to growth at all times $t$ is proportional to the mass of foam cells $M_{\mathrm{FC}}(t)$ at this time. Hence, we demand

$\Delta V_{\mathrm{Growth}}(t)=\alpha M_{\mathrm{FC}}(t)$,

where $\alpha$ is the proportionality constant and corresponds to the amount of volume occupied by a unit mass of foam cells, i.e. it is the inverse of the statistical mass density of foam cells. We can deduce

$V_{\mathrm{Growth}}(t)-V(0)=\alpha M_{\mathrm{FC}}(t)$

and express this in terms of integrals over the corresponding domains

$\int_{\Omega_{\text {Growth }}^{\mathcal{S}}(t)} 1 \mathrm{~d} \hat{V}-\int_{\Omega_{0}^{\mathcal{S}}} 1 \mathrm{~d} V=\alpha \int_{\Omega^{\mathcal{S}}(t)} c_{\mathrm{FC}}^{\mathcal{S} \mathcal{S}}(t) \mathrm{d} v$,

where $\mathrm{d} \hat{V}, \mathrm{~d} V$ and $\mathrm{d} v$ denote an integration over the corresponding growth, material and spatial configurations, respectively. We pull-back all integrals to the material configuration to achieve

$$
\int_{\Omega_{0}^{\mathcal{S}}} J_{\text {Growth }}^{\mathcal{S}}(t) \mathrm{d} V-\int_{\Omega_{0}^{\mathcal{S}}} 1 \mathrm{~d} V=\alpha \int_{\Omega_{0}^{\mathcal{S}}} J^{\mathcal{S}}(t) c_{\mathrm{FC}}^{\mathcal{S} \mathcal{S}}(t) \mathrm{d} V,
$$

where $J^{\mathcal{S}}(t)=\operatorname{det}\left(\mathbf{F}^{\mathcal{S}}(t)\right)$ and $J_{\text {Growth }}^{\mathcal{S}}(t)$ $=\operatorname{det}\left(\mathbf{F}_{\text {Growth }}^{\mathcal{S}}(t)\right) \stackrel{(13)}{=} \vartheta\left(c_{\mathrm{FC}}^{\mathcal{S}}(t)\right)$ are the Jacobian determinants of the deformation gradient $\mathbf{F}^{\mathcal{S}}$ and the growth part $\mathbf{F}_{\text {Growth }}^{\mathcal{S}}(t)$ of the deformation gradient at time $t$, respectively. Since (17) also holds locally we can conclude with a result similar to [56]:

$\vartheta\left(c_{\mathrm{FC}}^{\mathcal{S}}(t)\right)=1+\alpha J^{\mathcal{S}}(t) c_{\mathrm{FC}}^{\mathcal{S}}(t)$

In an atherosclerosis specific setup the unit radial direction ra at time $t$ is equal to the unit outer normal $\mathbf{n}_{\text {FS3I }}^{\mathcal{S}}(t)$ of the deformed surface $\Gamma_{\text {FS3I }}^{\mathcal{S}}(t)$. Hence, the radial direction does change due to the hemodynamics and preceded growth. Thus, equation (13) is not valid in an atherosclerotic context and we have to use an incremental definition of the growth part $\mathbf{F}_{\text {Growth }}^{\mathcal{S}}$ of the deformation gradient $[38,56]$. Let therefore be $t, \tau$ be instances in time with $\tau<t$, where in the interval $[\tau ; t]$ the growth direction can be assumed to be constant. Consequently, we can compute the growth part $\mathbf{F}_{\text {Growth }}^{\mathcal{S}}(t)$ of the deformation gradient at time $t$ by

$\mathbf{F}_{\text {Growth }}^{\mathcal{S}}(t)=\Delta \mathbf{F}_{\text {Growth }}^{\mathcal{S}}(\tau, t) \mathbf{F}_{\text {Growth }}^{\mathcal{S}}(\tau)$,

where $\mathbf{F}_{\text {Growth }}^{\mathcal{S}}(\tau)$ is the growth history part of the deformation gradient at time $\tau$ and $\Delta \mathbf{F}_{\text {Growth }}^{\mathcal{S}}(\tau, t)$ is the incremental growth deformation gradient from $\tau$ to $t$. The incremental growth deformation gradient is computed by

$$
\begin{aligned}
& \Delta \mathbf{F}_{\text {Growth }}^{\mathcal{S}}(\tau, t)=\mathbf{1} \\
& \quad+\frac{\vartheta\left(c_{\mathrm{FC}}^{\mathcal{S}}(t)\right)-\vartheta\left(c_{\mathrm{FC}}^{\mathcal{S S}}(\tau)\right)}{\vartheta\left(c_{\mathrm{FC}}^{\mathcal{S}}(\tau)\right)} \mathbf{n}_{\mathrm{FS} 3 \mathrm{I}}^{\mathcal{S}}(t) \otimes \mathbf{n}_{\mathrm{FS} 3 \mathrm{I}}^{\mathcal{S}}(t) .
\end{aligned}
$$

This incremental growth deformation gradient corresponds to a growth of the structure in the current radial direction $\mathbf{n}_{\text {FS3I }}^{\mathcal{S}}(t)$ by the factor $(\vartheta(t)-\vartheta(\tau)) / \vartheta(\tau)$ compared to the state at time $\tau$.

Remark. Iff the direction of growth is constant for all times $t$, then the incremental growth deformation gradient based formulation, i.e. equations (19) and (20) are equivalent to the representation in equation (13). 


\section{Remodeling and constitutive laws}

In the previous section, we have derived a growth model of the structure representing the increase of volume due to the deposition of foam cells. Along with growth also the change of mechanical properties of the structure is considered since foam cells feature a very different mechanical behavior compared to healthy aortic tissue. We follow the idea that with increased accumulation of foam cells the constitutive law of the structure locally and gradually changes to the one of foam cells. Hence, we compute the strain-energy density function $\Psi^{\mathcal{S}}$ of the hyperelastic structure as a convex combination of the strain-energy density function $\Psi_{\text {Ao }}^{\mathcal{S}}$ of healthy aortic tissue and the strain-energy density function $\Psi_{\text {FC }}^{\mathcal{S}}$ of pure foam cells

$\Psi^{\mathcal{S}}=\lambda\left(c_{\mathrm{FC}}^{\mathcal{S}}\right) \Psi_{\mathrm{Ao}}^{\mathcal{S}}+\left(1-\lambda\left(c_{\mathrm{FC}}^{\mathcal{S}}\right)\right) \Psi_{\mathrm{FC}}^{\mathcal{S}}$

where $\left.\left.\lambda\left(c_{\mathrm{FC}}^{\mathcal{S} \mathcal{S}}\right) \in\right] 0 ; 1\right]$ is the remodeling factor. It is a nonlinear function depending on the local concentration $c_{\mathrm{FC}}^{\mathcal{S}}$ and describes the ratio between the two extrema. To be more precise the remodeling factor $\lambda$ describes the fraction of volume of healthy aortic tissue compared to the overall (grown) volume. Since the change of overall volume relative to the initial volume is given by the growth factor $\vartheta\left(c_{\mathrm{FC}}^{\mathcal{S S}}\right)$ we can calculate the remodeling factor by

$\lambda\left(c_{\mathrm{FC}}^{\mathcal{S S}}\right)=\frac{1}{\vartheta\left(c_{\mathrm{FC}}^{\mathcal{S S}}\right)} \stackrel{(18)}{=} \frac{1}{1+\alpha J^{\mathcal{S}} c_{\mathrm{FC}}^{\mathcal{S S}}}$.

Consequently, at a position without foam cells, i.e. $c_{\mathrm{FC}}^{\mathcal{S S}}=$ 0 we get $\lambda=1$ resulting in healthy aortic material. In contrast, a large amount of foam cells, i.e. $c_{\mathrm{FC}}^{\mathcal{S S}} \rightarrow \infty$ results in $\lambda=0$ and hence in the mechanical properties that we assume for pure foam cells.

Since artery tissue is nearly incompressible [12,23], we use an additive split for both strain-energy functions into a volumetric and isochoric part [44,74]. For the specific choices of the volumetric parts $\Psi_{\text {Vol }}^{\mathcal{S}}$, see $[73$, $24]$. The artery wall can be seen as a ground material which is reinforced by fibers representing the collagen and elastin fibers. Hence, for the isochoric part of the healthy aortic tissue $\Psi_{\text {Ao }}^{\mathcal{S}}$ we exploit a four-fiber family model, see $[47,40,30,83]$

$$
\begin{aligned}
\Psi_{\text {Ao }}^{\mathcal{S}} & =\frac{c_{0, \text { Ao }}}{2}\left(\bar{I}_{\mathbf{C}^{\mathcal{S}}}-3\right) \\
& +\sum_{k=1}^{4} \frac{c_{1, k}}{4 c_{2, k}}\left(e^{\left(c_{2, k}\left(\left(\lambda_{k}\right)^{2}-1\right)^{2}\right)}-1\right),
\end{aligned}
$$

where the constants $c_{0, \mathrm{Ao}}, c_{1, k}$ and $c_{2, k}$ are aortic tissue specific material parameters. $\bar{I}_{\mathbf{C}^{\mathcal{S}}}=\left(J^{\mathcal{S}}\right)^{-2 / 3} \operatorname{tr}\left(\mathbf{C}^{\mathcal{S}}\right)$ is the first modified invariant of the right Cauchy-Green deformation tensor $\mathbf{C}^{\mathcal{S}}$ and $\lambda_{k}$ is the stretch of the $k$ th fiber family, respectively. Thereby, the stretch $\lambda_{k}$ is calculated by the total Cauchy-Green tensor $\mathbf{C}^{\mathcal{S}}$, see [86], and hence by $\lambda_{k}=\sqrt{\mathbf{M}_{k}^{T} \mathbf{C}^{\mathcal{S}} \mathbf{M}_{k}}$ where $\mathbf{M}_{k}=$ $\left[0, \sin \left(\delta_{k}\right), \cos \left(\delta_{k}\right)\right]^{T}$ is the direction of the $k$-th fiber in the radial, axial and circumferential coordinate system. The directions of the fibers are parametrized by the angles $\delta_{1}, \delta_{2}, \delta_{3}$ and $\delta_{4}$, which are material specific constants.

The mechanical behavior of atherosclerotic plaques is more comparable to a fluid than to a solid [68]. Therefore, a visco-hyperelastic Maxwell-like material, i.e. a spring and dashpot in series like approach is utilized as constitutive equation of foam cells [72,41,53,109]. The relaxation time of the viscous dashpot is $\tau_{\mathrm{FC}}[72]$ and for the isochoric part of the strain-energy density function $\Psi_{\mathrm{FC}}^{\mathcal{S}}$ we follow the idea of [5] using a modified neo-Hookean law

$\Psi_{\mathrm{FC}}^{\mathcal{S}}=\frac{c_{0, \mathrm{FC}}}{2}\left(\bar{I}_{\mathbf{C}^{\mathcal{S}}}-3\right)$,

where the constant $c_{0, \mathrm{FC}}$ is a material specific parameter.

Remark. If more species are assumed to induce the growth and remodeling of the artery wall, the presented laws can be generalized in a straightforward manner. The growth factor $\vartheta$ defined in equation (18) can be generalized to $\vartheta\left(\mathbf{c}^{\mathcal{S S}}\right)=1+J^{\mathcal{S}} \sum_{i} \alpha_{i} c_{i}^{\mathcal{S}}$, where $\mathbf{c}^{\mathcal{S S}}$ is the vector of all concentrations $c_{i}^{\mathcal{S} \mathcal{S}}$ of all growth inducing species $i$ and $\alpha_{i}$ are the corresponding growth parameters. The generalization for the remodeling process governed by equation $(22)$ reads $\Psi^{\mathcal{S}}=\frac{1}{\vartheta\left(\mathbf{c}^{\mathcal{S}}\right)} \Psi_{\text {Ao }}^{\mathcal{S}}$ $+\frac{J^{\mathcal{S}}}{\vartheta\left(\mathbf{c}^{\mathcal{S S}}\right)} \sum_{i} \alpha_{i} c_{i}^{\mathcal{S} \mathcal{S}^{\mathcal{S}}} \Psi_{i}^{\mathcal{S}}$, where the sum is again over all remodeling inducing species $i$.

\subsubsection{ALE mesh movement}

We model the ALE field as quasi-elastostatic structure on the domain $\Omega_{0}^{\mathcal{G}}$ [108]. Its interface deformation is governed by the structure's interface displacement field reading $\mathbf{d}^{\mathcal{G}}=\mathbf{d}^{\mathcal{S}}$ on $\Gamma_{\text {FS3I }}^{\mathcal{G}}$. Analogue to the structure field a zero Dirichlet condition and zero traction boundary conditions are prescribed at in- and outflow cross sections $\Gamma_{\text {In }}^{\mathcal{G}}$ and $\Gamma_{\text {Out }, i}^{\mathcal{G}}$, respectively.

\subsubsection{Fluid-structure interaction}

At the FS3I interface $\Gamma_{\text {FS3I }}$, we require kinematic continuity of fluid and structure velocity fields, i.e.

$\mathbf{u}^{\mathcal{F}}=\mathbf{u}^{\mathcal{S}}$ 
as well as the equilibrium of interface traction fields [61]

$\boldsymbol{\sigma}^{\mathcal{S}} \mathbf{n}_{\mathrm{FS} 3 \mathrm{I}}^{\mathcal{S}}=\mathbf{h}_{\mathrm{FS} 3 \mathrm{I}}^{\mathcal{S}}=-\mathbf{h}_{\mathrm{FS} 3 \mathrm{I}}^{\mathcal{F}}=-\boldsymbol{\sigma}^{\mathcal{F}} \mathbf{n}_{\mathrm{FS} 3 \mathrm{I}}^{\mathcal{S}}$,

where $\boldsymbol{\sigma}^{\mathcal{F}}$ and $\boldsymbol{\sigma}^{\mathcal{S}}$ are the Cauchy stress tensors of the fluid and structure, respectively. The kinematic constraint is enforced weakly via a Lagrange multiplier field $\boldsymbol{\Lambda}$, which allows for an interpretation of the Lagrange multiplier field as the interface traction. Here, we make the arbitrary choice $\boldsymbol{\Lambda}=\mathbf{h}_{\mathrm{FS} \text { II }}^{\mathcal{S}}$, i.e. the Lagrange multiplier field is seen as the interface traction acting onto the structure side of the interface $\Gamma_{\text {FS3I }}^{\mathcal{S}}$.

\subsection{Scalar concentrations of species}

All species are modeled by a continuum approach, i.e. we describe them as mass concentrations. The general framework of our simplified atherosclerosis model as described in section 2.1 is given by the advection-diffusion-reaction equation $[58,65,99]$. The LDL transport in the lumen is dominated by advection, whereas in the artery wall it is assumed to be solely driven by diffusion. In addition, in the artery wall species are produced and degraded by biochemical reactions. The complex heterogeneous structure of the artery wall is currently neglected and we utilize a so-called fluid-wall model $[110,81]$, where the endothelium is considered to be the only transport barrier. The endothelium acts as a semi-permeable membrane leading to a significant discontinuity between the concentrations in the blood and in the artery wall. Therefore, the calculation of LDL is divided into two separate, but coupled domains: the domain of the scalar-valued concentration in the fluid $\Omega^{\mathcal{F S}}$ (fluid-scalar) and the domain of scalar-valued concentrations in the structure $\Omega^{\mathcal{S S}}$ (structure-scalars). The domains $\Omega^{\mathcal{F S}}$ and $\Omega^{\mathcal{S S}}$ match the domains of the fluid $\Omega^{\mathcal{F}}$ and the structure $\Omega^{\mathcal{S}}$, respectively. Still we denote the corresponding quantities with $\mathcal{F} \mathcal{S}$ and $\mathcal{S} \mathcal{S}$ to easily distinguish between the fluid, structure, fluidscalar and structure-scalar quantities.

\subsubsection{Concentrations in the blood}

The transportation of the mass concentration of LDL with the blood flow is modeled by the advection-diffusion equation. Hence, the dynamic of the scalar-valued concentration $c_{\mathrm{LDL}}^{\mathcal{F} \mathcal{S}}$ of LDL inside the deformable fluidscalar domain $\Omega^{\mathcal{F S}}(t)$ is described by

$$
\begin{aligned}
\left.\frac{\partial}{\partial t} c_{\mathrm{LDL}}^{\mathcal{F} \mathcal{S}}\right|_{\chi} & +\left(\mathbf{u}^{\mathcal{F}}-\mathbf{u}^{\mathcal{G}}\right) \cdot \nabla c_{\mathrm{LDL}}^{\mathcal{F} \mathcal{S}} \\
& -\nabla \cdot\left(D_{\mathrm{LDL}}^{\mathcal{F} \mathcal{S}} \nabla c_{\mathrm{LDL}}^{\mathcal{F} \mathcal{S}}\right)=0,
\end{aligned}
$$

in an ALE observer frame. The motion of the ALE observer is the same as for the fluid field, see section 2.3.4.
The constant $D_{\mathrm{LDL}}^{\mathcal{F} \mathcal{S}}$ is the diffusivity of LDL in blood. On the inflow boundary $\Gamma_{\text {In }}^{\mathcal{F} \mathcal{S}}$ we apply a Dirichlet condition:

$c_{\mathrm{LDL}}^{\mathcal{F S}}=c_{\mathrm{LDL}, \mathrm{In}}^{\mathcal{F} \mathcal{S}}$

On the outflow boundaries $\Gamma_{\text {Out }, i}^{\mathcal{F} \mathcal{S}}$ we use the symmetry condition

$\nabla c_{\mathrm{LDL}}^{\mathcal{F S}} \cdot \mathbf{n}_{\text {Out }, i}^{\mathcal{F S}}=0$

The flux of LDL through the FS3I interface $\Gamma_{\text {FS3I }}^{\mathcal{F S}}$, i.e. the endothelium is described by

$$
\left(-D_{\mathrm{LDL}}^{\mathcal{F} \mathcal{S}} \nabla c_{\mathrm{LDL}}^{\mathcal{F S}}+\left(\mathbf{u}^{\mathcal{F}}-\mathbf{u}^{\mathcal{G}}\right) c_{\mathrm{LDL}}^{\mathcal{F S}}\right) \cdot \mathbf{n}_{\mathrm{FS} 3 \mathrm{I}}^{\mathcal{F} \mathcal{S}}=-J_{\mathrm{Sol}}\left(c_{\mathrm{LDL}}\right),
$$

where $J_{\text {Sol }}\left(c_{\mathrm{LDL}}^{\mathcal{F} \mathcal{S}}\right)$ is the solute flux and is described in section 2.4.3. It is important to note that we have assumed the artery wall is a solid. Hence, using equation (25) reduces the flux condition to:

$-D_{\mathrm{LDL}}^{\mathcal{F} \mathcal{S}} \nabla c_{\mathrm{LDL}}^{\mathcal{F} \mathcal{S}} \cdot \mathbf{n}_{\mathrm{FS} 3 \mathrm{I}}^{\mathcal{F} \mathcal{S}}=-J_{\mathrm{Sol}}\left(c_{\mathrm{LDL}}\right)$.

\subsubsection{Concentrations in the artery wall}

The transport and interaction of species in the artery wall is modeled by the diffusion-reaction equation. Hence, the dynamic of the concentration $c_{\mathrm{LDL}}^{\mathcal{S}}$ of LDL in the deforming structure-scalars domain $\Omega^{\mathcal{S}}(t)$ is described by

$$
\begin{aligned}
\left.\frac{\partial}{\partial t} c_{\mathrm{LDL}}^{\mathcal{S S}}\right|_{\chi} & +c_{\mathrm{LDL}}^{\mathcal{S S}} \nabla \cdot \mathbf{u}^{\mathcal{S}} \\
& -\boldsymbol{\nabla} \cdot\left(D_{\mathrm{LDL}}^{\mathcal{S S}} \nabla c_{\mathrm{LDL}}^{\mathcal{S S}}\right)-r_{\mathrm{LDL}}^{\mathcal{S S}}\left(\mathbf{c}^{\mathcal{S S}}\right)=0,
\end{aligned}
$$

in an ALE observer frame. The motion of the arbitrary observer is given by the structure field (see section 2.3.3) and its velocity field is $\mathbf{u}^{\mathcal{S}}$. The constant $D_{\mathrm{LDL}}^{\mathcal{S} \mathcal{S}}$ is the diffusivity of LDL in the artery wall. The reaction term $r_{\mathrm{LDL}}^{\mathcal{S S}}\left(\mathbf{c}^{\mathcal{S S}}\right)$ is a function depending on the concentrations $\mathbf{c}^{\mathcal{S}}$ of all species considered in the artery wall.

We restrict ourself to a simplistic model of the atherosclerotic process in the artery wall. It considers two species only: LDL and foam cells. Thereby LDL does not exclusively model low-density lipoproteins but represents more general all species involved in the inflammatory processes such as LDL, radical oxygen species, high-density lipoproteins or modified LDL. Foam cells represent the final products of the complex biochemical processes like monocytes, macrophages, smooth muscle cells, foam cells and others. Inside the domain $\Omega^{\mathcal{S S}}$ the concentration of foam cells $c_{\mathrm{FC}}^{\mathcal{S}}$ is in analogy to equation (32).

We assume that there are healing processes resulting in the degradation of the scalar-valued quantity $c_{\mathrm{LDL}}^{\mathcal{S S}}$. 
Furthermore, foam cells are produced if the concentration of LDL $c_{\mathrm{LDL}}^{\mathcal{S S}}$ exceeds a given threshold $c_{\mathrm{LDL}}^{\mathcal{S} \text {, Thres }}$. Hence, the reactive term of LDL is

$r_{\mathrm{LDL}}^{\mathcal{S S}}\left(\mathbf{c}^{\mathcal{S S}}\right)=-d_{\mathrm{LDL}}^{\mathcal{S S}} c_{\mathrm{LDL}}^{\mathcal{S S}}-\gamma_{\mathrm{LDL}}^{\mathcal{S S}}\left(c_{\mathrm{LDL}}^{\mathcal{S S}}-c_{\mathrm{LDL}, \text { Thres }}^{\mathcal{S S}}\right)_{+}$,

where the constants $d_{\mathrm{LDL}}^{\mathcal{S} \mathcal{S}}$ and $\gamma_{\mathrm{LDL}}^{\mathcal{S} \mathcal{S}}$ are the degradation and reaction rate of LDL, respectively. The index $(\star)_{+}$ denotes the positive branch of $(\star)$, i.e. it is zero when its argument is negative. Foam cells are a product of LDL and are not degraded. The reactive term of foam cells reads

$r_{\mathrm{FC}}^{\mathcal{S S}}\left(\mathbf{c}^{\mathcal{S S}}\right)=\gamma_{\mathrm{LDL}}^{\mathcal{S S}}\left(c_{\mathrm{LDL}}^{\mathcal{S S}}-c_{\mathrm{LDL}, \text { Thres }}^{\mathcal{S S}}\right)_{+}$.

On the boundaries $\Gamma_{\mathrm{In}}^{\mathcal{S S}}$ and $\Gamma_{\text {Out }, i}^{\mathcal{S} \mathcal{S}}$ we use the symmetry conditions

$$
\begin{gathered}
\nabla c_{\mathrm{LDL}}^{\mathcal{S S}} \cdot \mathbf{n}_{\mathrm{In}}^{\mathcal{S S}}=0=\nabla c_{\mathrm{LDL}}^{\mathcal{S S}} \cdot \mathbf{n}_{\mathrm{Out}, i}^{\mathcal{S S}} \\
\nabla c_{\mathrm{FC}}^{\mathcal{S S}} \cdot \mathbf{n}_{\mathrm{In}}^{\mathcal{S S}}=0=\nabla c_{\mathrm{FC}}^{\mathcal{S S}} \cdot \mathbf{n}_{\mathrm{Out}, i}^{\mathcal{S S}} .
\end{gathered}
$$

We assume the artery wall to be impervious at its outer boundary $\Gamma_{\text {Wall }}^{\mathcal{S} \mathcal{S}}$ and hence no flux conditions

$$
\begin{array}{r}
-D_{\mathrm{LDL}}^{\mathcal{S S}} \nabla c_{\mathrm{LDL}}^{\mathcal{S S}} \cdot \mathbf{n}_{\text {Wall }}^{\mathcal{S S}}=0 \\
-D_{\mathrm{FC}}^{\mathcal{S S}} \nabla c_{\mathrm{FC}}^{\mathcal{S S}} \cdot \mathbf{n}_{\text {Wall }}^{\mathcal{S S}}=0
\end{array}
$$

are imposed. The diffusive influx of LDL through $\Gamma_{\text {FS3I }}^{\mathcal{S S}}$, i.e. the endothelium is given by

$-D_{\mathrm{LDL}}^{\mathcal{S S}} \nabla c_{\mathrm{LDL}}^{\mathcal{S S}} \cdot \mathbf{n}_{\mathrm{FS} 3 \mathrm{I}}^{\mathcal{S S}}=J_{\mathrm{Sol}}\left(c_{\mathrm{LDL}}\right)$

whereas foam cells can not migrate through the endothelium:

$-D_{\mathrm{FC}}^{\mathcal{S S}} \nabla c_{\mathrm{FC}}^{\mathcal{S S}} \cdot \mathbf{n}_{\mathrm{FS} 3 \mathrm{I}}^{\mathcal{S S}}=0$

Remark. It is highlighted again that the present model neglects the advective transport of LDL through the endothelium and inside the artery wall driven by transmural pressure gradients. To consider these effects either a full fluid-porous-structure interaction approach must be chosen for the cardiovascular mechanics or the presented model has to be enriched by a flow model on the structure domain as in [58].

\subsubsection{Kedem-Katchalsky equations and wall-shear stress modulated permeability}

The endothelium is frequently modeled as semi-permeable membrane described by the equations of Kedem and Katchalsky [55,95,106,11,54,46,81]. We have assumed the artery wall to be a pure solid and hence the second
Kedem-Katchalsky equation describing the solute fluxes reduces to $[11,46]$

$J_{\mathrm{Sol}}\left(c_{\mathrm{LDL}}\right)=P_{D}\left(c_{\mathrm{LDL}}^{\mathcal{F S}}-c_{\mathrm{LDL}}^{\mathcal{S S}}\right)$

where $P_{D}$ is the diffusive permeability of the endothelium. This neglection of the convective mass transport through the endothelium lies in agreement with observations in literature $[97,46]$. It is well-accepted that the localization of atherosclerosis correlates with hemodynamic factors such as low wall-shear stresses [80,82,59, $3,43]$. The wall-shear stresses ${ }^{1} \boldsymbol{\tau}^{\mathcal{F}}$ of the fluid acting on the FS3I interface $\Gamma_{\mathrm{FS} 3 \mathrm{I}}$, i.e. the endothelium, are calculated by removing the normal parts of the tractions

$\boldsymbol{\tau}^{\mathcal{F}}=\boldsymbol{\sigma}^{\mathcal{F}} \mathbf{n}_{\mathrm{FS} 3 \mathrm{I}}^{\mathcal{F}}-\left(\left(\mathbf{n}_{\mathrm{FS} 3 \mathrm{I}}^{\mathcal{F}}\right)^{T} \boldsymbol{\sigma}^{\mathcal{F}} \mathbf{n}_{\mathrm{FS} 3 \mathrm{I}}^{\mathcal{F}}\right) \mathbf{n}_{\mathrm{FS} 3 \mathrm{I}}^{\mathcal{F}}$

The WSS dependency of the endothelium is on a much larger time scale than the cardiovascular mechanics. It is considered by adapting the diffusive permeability $P_{D}$ by a function $s$ depending on the norm of the time-averaged $\left.\mathrm{WSS}<\boldsymbol{\tau}^{\mathcal{F}}\right\rangle_{t}$

$J_{\mathrm{Sol}}\left(c_{\mathrm{LDL}}, \boldsymbol{\tau}^{\mathcal{F}}\right)=P_{D} s\left(\left\|<\boldsymbol{\tau}^{\mathcal{F}}>_{t}\right\|\right)\left(c_{\mathrm{LDL}}^{\mathcal{F} \mathcal{S}}-c_{\mathrm{LDL}}^{\mathcal{S S}}\right)$

where time-average of the WSS $\boldsymbol{\tau}^{\mathcal{F}}$ at time $t$ is defined as

$<\boldsymbol{\tau}^{\mathcal{F}}>_{t}=\frac{1}{T_{\mathrm{Cycl}}} \int_{t-T_{\mathrm{Cycl}}}^{t} \boldsymbol{\tau}^{\mathcal{F}}(s) \mathrm{d} s$.

Remark. One could also include other hemodynamic factors like the oscillatory shear index (OSI) or the relative residence time $[43,80,89]$ into the calculation of $s(\star)$.

We call $\left.s\left(\|<\boldsymbol{\tau}^{\mathcal{F}}\right\rangle_{t} \|\right)$ the permeability scaling factor (PSF). For the shape of the PSF $s$ we follow the idea in [11]:

$s\left(\left\|<\boldsymbol{\tau}^{\mathcal{F}}>_{t}\right\|\right)=\frac{1}{\ln (2)} \ln \left(1+\frac{m_{1}}{\left\|<\boldsymbol{\tau}^{\mathcal{F}}>_{t}\right\|+m_{2}}\right)$,

where the constants $m_{1}$ and $m_{2}$ are free model parameters which have to be fitted to the specific geometry, see section 4.1. The PSF is a monotonically decreasing function with respect to WSS resulting in an increased permeability of the endothelium with respect to LDL at regions of low WSS.

1 Wall-shear stresses is the esablished name even though wall-shear tractions would be more acurate 
2.5 Initial conditions and prestressing

To achieve a well-defined initial value problem the specific initial conditions are stated. For the FSI part we use zero initial conditions and smoothly increase the prescribed fluid influx $Q_{\text {In }}^{\mathcal{F}}(t)$ to its physiological level. We aim at utilizing geometries stemming from in vivo medical imaging that do not represent a stress-free configuration. We therefore apply prestressing according to [35] for the structure field. Therein, the Windkessel pressure on the outflow boundaries does lead to a physiological diastolic pressure of the fluid field and hence to physiological loading of the structure comparable to the in vivo state.

For the concentrations in the artery wall zero initial conditions are prescribed. For initial condition of the concentration in the blood, constant concentrations equal to values prescribed on the inflow boundary $\Gamma_{\text {In }}^{\mathcal{F} \mathcal{S}}$ are utilized.

\section{Numerical procedure}

For computationally solving the model the weak form is established, its spatial and temporal discretization is performed and stability issues arising in the advection dominated fields are dealt with. Additionally, an appropriate strategy for solving the strongly coupled discrete problem is introduced, resulting in large linear systems solved by suitable methods. The following gives a brief overview of the utilized methods without going into detail. All implementations have been done in the multiphysics framework BACI [100].

\subsection{Weak form}

The establishment of the weak form of the model from the strong equations requires the definition of appropriate solution spaces $\mathcal{S}$ and trial spaces $\mathcal{T}$ for all fields:

$$
\begin{aligned}
& \mathcal{S}_{\mathbf{u}^{\mathcal{F}}}=\left\{\mathbf{u}^{\mathcal{F}} \in\left(\mathcal{H}^{1}\left(\Omega^{\mathcal{F}}\right)\right)^{3} \mid\right. \\
& \left.\mathbf{u}^{\mathcal{F}}=-Q_{\text {In }}^{\mathcal{F}} g \mathbf{n}_{\text {In }}^{\mathcal{F}} \text { on } \Gamma_{\text {In }}^{\mathcal{F}}\right\} \\
& \mathcal{S}_{p^{\mathcal{F}}}=\left\{p^{\mathcal{F}} \in \mathcal{L}^{2}\left(\Omega^{\mathcal{F}}\right)\right\} \\
& \mathcal{S}_{\mathbf{d}^{\mathcal{S}}}=\left\{\mathbf{d}^{\mathcal{S}} \in\left(\mathcal{H}^{1}\left(\Omega^{\mathcal{S}}\right)\right)^{3} \mid \mathbf{d}^{\mathcal{S}}=\mathbf{0} \text { on } \Gamma_{\operatorname{In}}^{\mathcal{S}}\right\} \\
& \mathcal{S}_{\mathbf{d}^{\mathcal{G}}}=\left\{\mathbf{d}^{\mathcal{G}} \in\left(\mathcal{H}^{1}\left(\Omega^{\mathcal{G}}\right)\right)^{3} \mid \mathbf{d}^{\mathcal{G}}=\mathbf{0} \text { on } \Gamma_{\operatorname{In}}^{\mathcal{G}}\right\} \\
& \mathcal{S}_{\boldsymbol{\Lambda}}=\left\{\boldsymbol{\Lambda} \in\left(\mathcal{H}^{-\frac{1}{2}}\left(\Gamma_{\mathrm{FS} 3 \mathrm{I}}\right)\right)^{3}\right\} \\
& \mathcal{S}_{c^{\mathcal{F} S}}=\left\{c^{\mathcal{F S}} \in \mathcal{H}^{1}\left(\Omega^{\mathcal{F S}}\right) \mid c^{\mathcal{F S}}=c_{\mathrm{In}}^{\mathcal{F} \mathcal{S}} \text { on } \Gamma_{\mathrm{In}}^{\mathcal{F} \mathcal{S}}\right\} \\
& \mathcal{S}_{c} \mathcal{S} \mathcal{S}=\left\{c^{\mathcal{S S}} \in \mathcal{H}^{1}\left(\Omega^{\mathcal{S S}}\right)\right\} \text {, }
\end{aligned}
$$

where $\mathcal{H}^{1}\left(\Omega^{(\star)}\right), \mathcal{L}^{2}\left(\Omega^{(\star)}\right)$ and $\mathcal{H}^{-\frac{1}{2}}\left(\Gamma_{(\star)}\right)$ are the usual Sobolev spaces. The trial spaces $\mathcal{T}$ are equal to the corresponding solution spaces $\mathcal{S}$, but with homogeneous Dirichlet conditions. The overall weak subproblem of the FSI model of the cardiovascular mechanics reads: Find

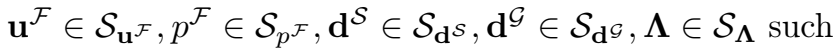
that [70]:

$$
\begin{aligned}
& 0=r^{\mathcal{F}}=\left(\delta \mathbf{u}^{\mathcal{F}},\left.\varrho^{\mathcal{F}} \frac{\partial}{\partial t} \mathbf{u}^{\mathcal{F}}\right|_{\chi}\right)_{\Omega^{\mathcal{F}}} \\
& +\left(\delta \mathbf{u}^{\mathcal{F}}, \varrho^{\mathcal{F}}\left(\left(\mathbf{u}^{\mathcal{F}}-\mathbf{u}^{\mathcal{G}}\right) \cdot \nabla\right) \mathbf{u}^{\mathcal{F}}\right)_{\Omega^{\mathcal{F}}} \\
& +\left(\nabla \delta \mathbf{u}^{\mathcal{F}}, 2 \eta^{\mathcal{F}}\left(\mathbf{u}^{\mathcal{F}}\right) \varepsilon\left(\mathbf{u}^{\mathcal{F}}\right)\right)_{\Omega^{\mathcal{F}}} \\
& -\left(\boldsymbol{\nabla} \cdot \delta \mathbf{u}^{\mathcal{F}}, p^{\mathcal{F}}\right)_{\Omega^{\mathcal{F}}}-\left(\delta p^{\mathcal{F}}, \boldsymbol{\nabla} \cdot \mathbf{u}^{\mathcal{F}}\right)_{\Omega^{\mathcal{F}}} \\
& +\left(\delta \mathbf{u}^{\mathcal{F}}, p_{\mathrm{WK}, i}^{\mathcal{F}} \mathbf{n}_{\mathrm{Out}, i}^{\mathcal{F}}\right)_{\Gamma_{\mathrm{Out}, i}^{\mathcal{F}}}+\left(\delta \mathbf{u}^{\mathcal{F}}, \boldsymbol{\Lambda}\right)_{\Gamma_{\mathrm{FS} 3 \mathrm{I}}^{\mathcal{F}}}, \\
& 0=r^{\mathcal{S}}=\left(\delta \mathbf{d}^{\mathcal{S}}, \varrho_{0}^{\mathcal{S}} \frac{\mathrm{d}^{2}}{\mathrm{~d} t^{2}} \mathbf{d}^{\mathcal{S}}\right)_{\Omega^{\mathcal{S}}} \\
& +\left(\nabla \delta \mathbf{d}^{\mathcal{S}}, \mathbf{F}^{\mathcal{S}} \mathbf{S}^{\mathcal{S}}\left(\mathbf{C}^{\mathcal{S}}, c_{\mathrm{FC}}^{\mathcal{S}}\right)\right)_{\Omega^{\mathcal{S}}} \\
& +\left(\delta \mathbf{d}^{\mathcal{S}}, k_{\text {Wall }}^{\mathcal{S}} \mathbf{d}^{\mathcal{S}}+c_{\text {Wall }}^{\mathcal{S}} \frac{\mathrm{d}}{\mathrm{d} t} \mathbf{d}^{\mathcal{S}}\right)_{\Gamma_{\text {Wall }}^{\mathcal{S}}} \\
& +\left(\delta \mathbf{d}^{\mathcal{S}}, \mathbf{N}_{\text {Out }, i}^{\mathcal{S}} \cdot\left(k_{\text {Out }}^{\mathcal{S}} \mathbf{d}^{\mathcal{S}}+\right.\right. \\
& \left.\left.c_{\mathrm{Out}}^{\mathcal{S}} \frac{\mathrm{d}}{\mathrm{d} t} \mathbf{d}^{\mathcal{S}}\right) \mathbf{N}_{\mathrm{Out}, i}^{\mathcal{S}}\right)_{\Gamma_{\mathrm{Out}, i}^{\mathcal{S}}} \\
& -\left(\delta \mathbf{d}^{\mathcal{S}}, \boldsymbol{\Lambda}\right)_{\Gamma_{\mathrm{FS} 3 \mathrm{I}}^{\mathcal{S}}}, \\
& 0=r^{\text {coupl }}=\left(\delta \boldsymbol{\Lambda}, \mathbf{d}^{\mathcal{S}}-\mathbf{d}^{\mathcal{G}}\right)_{\Gamma_{\mathrm{FS} 3 \mathrm{I}}^{\mathcal{S}}},
\end{aligned}
$$

for all $\delta \mathbf{u}^{\mathcal{F}} \in \mathcal{T}_{\mathbf{u}^{\mathcal{F}}}, \delta p^{\mathcal{F}} \in \mathcal{T}_{p^{\mathcal{F}}}, \delta \mathbf{d}^{\mathcal{S}} \in \mathcal{T}_{\mathbf{d}^{\mathcal{S}}}, \delta \mathbf{d}^{\mathcal{G}} \in \mathcal{T}_{\mathbf{d}^{\mathcal{G}}}$ and $\delta \boldsymbol{\Lambda} \in \mathcal{T}_{\boldsymbol{\Lambda}}$. Thereby, $(\star, \star)_{\Omega^{(\star)}}$ and $(\star, \star)_{\Gamma^{(\star)}}$ denote the usual $L^{2}$ inner products on $\Omega^{(\star)}$ and $\Gamma^{(\star)}$, respectively. The overall weak subproblem of the SSI model for the scalar concentrations of species reads: Find $c_{i}^{\mathcal{F} \mathcal{S}} \in \mathcal{S}_{c^{\mathcal{F} S}}$ and $c_{i}^{\mathcal{S S}} \in \mathcal{S}_{c^{\mathcal{S S}}}$ such that [108]:

$$
\begin{aligned}
& 0=r_{i}^{\mathcal{F} \mathcal{S}}=\left(\delta c_{i}^{\mathcal{F} \mathcal{S}},\left.\frac{\partial}{\partial t} c_{i}^{\mathcal{F} \mathcal{S}}\right|_{\chi}\right)_{\Omega^{\mathcal{F} \mathcal{S}}} \\
& +\left(\delta c_{i}^{\mathcal{F S}},\left(\mathbf{u}^{\mathcal{F}}-\mathbf{u}^{\mathcal{G}}\right) \cdot \nabla c_{i}^{\mathcal{F S}}\right)_{\Omega^{\mathcal{F} S}} \\
& +\left(\nabla \delta c_{i}^{\mathcal{F S}}, D_{i}^{\mathcal{F} \mathcal{S}} \nabla c_{i}^{\mathcal{F} \mathcal{S}}\right)_{\Omega^{\mathcal{F} S}} \\
& -\left(\delta c_{i}^{\mathcal{F} \mathcal{S}}, J_{\mathrm{Sol}}\left(c_{i}, \boldsymbol{\tau}^{\mathcal{F}}\right)\right)_{\Gamma_{\mathrm{FS} 3 \mathrm{I}}^{\mathcal{F} \mathcal{S}}}, \\
& 0=r_{i}^{\mathcal{S S}}=\left(\delta c_{i}^{\mathcal{S S}},\left.\frac{\partial}{\partial t} c_{i}^{\mathcal{S S}}\right|_{\chi}\right)_{\Omega^{\mathcal{S S}}} \\
& -\left(\delta c_{i}^{\mathcal{S S}}, c_{i}^{\mathcal{S S}} \nabla \cdot \mathbf{u}^{\mathcal{S}}\right)_{\Omega^{\mathcal{S S}}} \\
& +\left(\nabla \delta c_{i}^{\mathcal{S S}}, D_{i}^{\mathcal{S S}} \nabla c_{i}^{\mathcal{S} \mathcal{S}}\right)_{\Omega^{\mathcal{S S}}} \\
& -\left(\delta c_{i}^{\mathcal{S S}}, r_{i}^{\mathcal{S S}}\left(\mathbf{c}^{\mathcal{S S}}\right)\right)_{\Omega^{\mathcal{S S}}} \\
& +\left(\delta c_{i}^{\mathcal{S S}}, J_{\mathrm{Sol}}\left(c_{i}, \boldsymbol{\tau}^{\mathcal{F}}\right)\right)_{\Gamma_{\mathrm{FS} 3 \mathrm{I}}^{\mathcal{S}}},
\end{aligned}
$$

for all $\delta c_{i}^{\mathcal{F S}} \in \mathcal{T}_{c^{\mathcal{F} S}}, \delta c_{i}^{\mathcal{S S}} \in \mathcal{T}_{c^{\mathcal{S}}}$ and $i=\mathrm{LDL}, \mathrm{FC}$. 


\subsection{Discretization}

A Galerkin Finite Element approximation of the weak form is performed. Therefore, the domains $\Omega^{\mathcal{F}}$ and $\Omega^{\mathcal{S}}$ are discretized using $n^{\mathcal{F}}$ and $n^{\mathcal{S}}$ elements, respectively. The remaining domains $\Omega^{\mathcal{G}}, \Omega^{\mathcal{F S}}$ and $\Omega^{\mathcal{S}}$ match either $\Omega^{\mathcal{F}}$ or $\Omega^{\mathcal{S}}$ and utilize the same meshes. To obtain the semi-discrete problem the spatial discretization is performed by means of the finite element method with Lagrange polynomials as shape and ansatz functions.

The spatially discrete but temporal continuous, nonlinear coupled problem for the vector $\mathbf{y}(t)=\left[\mathbf{u}_{h}^{\mathcal{F}}, p_{h}^{\mathcal{F}}, \mathbf{d}_{h}^{\mathcal{S}}, \mathbf{d}_{h}^{\mathcal{G}}, \boldsymbol{\Lambda}_{h}, \mathbf{c}_{h}^{\mathcal{F} \mathcal{S}}, \mathbf{c}_{h}^{\mathcal{S} \mathcal{S}}\right]^{T}$ of the nodal ansatz coefficients can be written in the form

$\frac{\mathrm{d}}{\mathrm{d} t} \mathbf{y}(t)-\mathbf{f}(t, \mathbf{y}(t))=\mathbf{0}$,

where the nonlinear function $\mathbf{f}$ corresponds to the spatially discrete version of the model. For the discretization in time, the one-step- $\theta$ scheme is exploited to achieve an approximating sequence $\left\{\mathbf{y}^{n}\right\}_{n=0,1}$ of the time continuous problem by finding the root of the discrete residual $\mathbf{r}$ :

$$
\begin{aligned}
\mathbf{r}\left(t^{n+1}, \mathbf{y}^{n+1}\right)= & \mathbf{y}^{n+1}-\mathbf{y}^{n}-\Delta t\left(\theta \mathbf{f}\left(t^{n+1}, \mathbf{y}^{n+1}\right)\right. \\
& \left.+(1-\theta) \mathbf{f}\left(t^{n}, \mathbf{y}^{n}\right)\right)=\mathbf{0}
\end{aligned}
$$

for each $n=0,1, \ldots, n_{T}$, where $t^{n}=n \Delta t$ and $\mathbf{y}^{n}=$ $\mathbf{y}\left(t^{n}\right)$. The time step size is denoted by $\Delta t$ and $n_{T}$ time steps are performed. The scheme coefficient $\theta$ is chosen to be $\theta=0.5$.

To overcome numerical stability issues arising from the spatial, equal-order Finite Element discretization, stabilization terms are added to the discrete fluid residual $\mathbf{r}^{\mathcal{F}}$. Namely we utilize the streamline-upwind PetrovGalerkin (SUPG), pressure-stabilized Petrov-Galerkin (PSPG) and grad-div stabilization, see $[27,76]$ and therein. The stabilization parameter is chosen according to [6]. Further, an additional backflow stabilization on each of the outflow boundaries $\Gamma_{\text {Out }, i}^{\mathcal{F}}$ is applied due to the pulsatile flow pattern enabling spontaneous backflows at these Neumann boundaries [39].

To stabilize the advection dominated fluid-scalar field we utilize the Galerkin least-squares method. Additionally, the $Y Z \beta$ discontinuity-capturing is applied $[7,54]$ to also resolve the steep concentration gradients occurring near the FS3I interface [62]. The stabilization parameter is chosen according to [18].

\subsection{FS3I solver strategy}

A suitable solver strategy is exploited taking into account the specific couplings between the individual fields. Due to the strong coupling between the fluid, structure and
ALE field by the FSI coupling conditions the strongly coupled FSI subproblem is addressed by a monolithic approach. The (fluid-scalar)-(structure-scalar) interaction (SSI) subproblem is solved monolithically too to account for the strong SSI interface coupling. FSI and SSI subproblems are only coupled in terms of growth and remodeling induced by foam cell taking place on a much larger time scale compared to the time scale of the FSI subproblem. Hence, the natural choice for solving the overall FS3I problem is by a sequentially staggered scheme coupling the monolithic FSI with the monolithic SSI problem. A schematic overview of the solver strategy including the corresponding coupling variables is given in Figure 3.

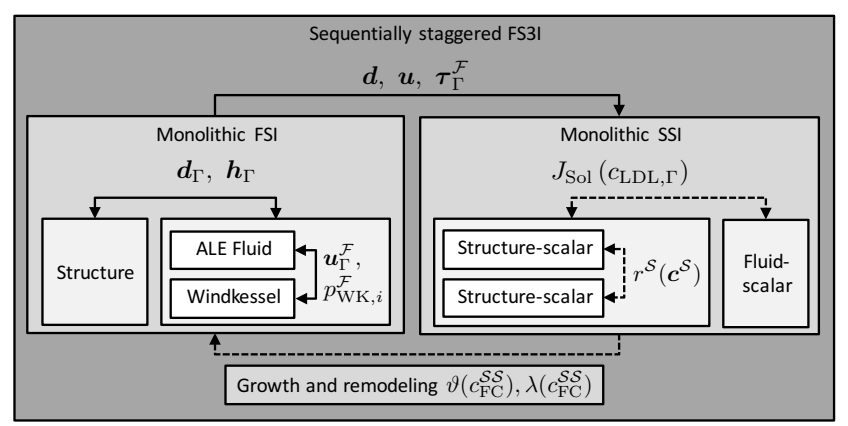

Fig. 3 Overview of the solver strategy for the presented fluid-structure-scalar-scalar interaction (FS3I) model including the coupling variables between the fields. Simple and double arrows mark one-way and two-way couplings, respectively. Dotted arrows denote weak couplings, whereas solid arrows represent strong couplings. The subindex $(\star)_{\Gamma}$ indicates a surface coupled quantity $(\star)$, whereas without explicit subindex a volume coupled quantity is denoted.

\subsection{Monolithic FSI}

The monolithic FSI problem to solve for the incremental structure displacements $\Delta \mathbf{d}_{h}^{\mathcal{S}}$, fluid velocities $\Delta \mathbf{u}_{h}^{\mathcal{F}}$ and Lagrange multipliers $\Delta \boldsymbol{\Lambda}_{h}$ reads [70]

$$
\begin{gathered}
\left(\begin{array}{ccc}
\frac{\partial}{\partial \mathbf{d}_{h}^{\mathcal{S}}} \mathbf{r}_{h}^{\mathcal{S}} & \mathbf{0} & \frac{\partial}{\partial \boldsymbol{\Lambda}_{h}} \mathbf{r}_{h}^{\mathcal{S}} \\
\mathbf{0} & \frac{\partial}{\partial \mathbf{u}_{h}^{\mathcal{F}}} \mathbf{r}_{h}^{\mathcal{F}} & \frac{\partial}{\partial \boldsymbol{\Lambda}_{h}} \mathbf{r}_{h}^{\mathcal{F}} \\
\frac{\partial}{\partial \mathbf{d}_{h}^{\mathcal{S}}} \mathbf{r}_{h}^{\operatorname{coupl}} & \frac{\partial}{\partial \mathbf{u}_{h}^{\mathcal{F}}} \mathbf{r}_{h}^{\text {coupl }} & \mathbf{0}
\end{array}\right)_{i}\left(\begin{array}{c}
\Delta \mathbf{d}_{h}^{\mathcal{S}} \\
\Delta \mathbf{u}_{h}^{\mathcal{F}} \\
\Delta \boldsymbol{\Lambda}_{h}
\end{array}\right)_{i+1}^{n+1} \\
=-\left(\begin{array}{c}
\mathbf{r}_{h}^{\mathcal{S}} \\
\mathbf{r}_{h}^{\mathcal{F}} \\
\mathbf{r}_{h}^{\text {coupl }}
\end{array}\right)_{i}^{n+1}
\end{gathered}
$$

where $n=0,1, \ldots, n_{T}$ is the time step and $i=0,1, \ldots$ is the Newton step. $\mathbf{r}_{h}^{\mathcal{S}}, \mathbf{r}_{h}^{\mathcal{F}}$ and $\mathbf{r}_{h}^{\text {coupl }}$ denote the spatial and temporal discrete non-linear residuals of the structure, fluid and FSI coupling, respectively. To ease the 
notation fluid pressure and ALE displacement degrees of freedom are merged together with the fluid velocities. The Newton iteration for time step $n$ is stopped if all 2norms $\left\|\mathbf{r}^{(\star)}\right\|_{2}$ of the individual single field residuals $\mathbf{r}^{(\star)}$ scaled to their lengths are below $10^{-5}$.

To resolve the coupling of the fluid with the multiple three-element windkessel models an analytic relationship $p_{\mathrm{WK}, i}^{\mathcal{F}}\left(Q_{\mathrm{Out}, i}^{\mathcal{F}}\right)$ between the windkessel pressures $p_{\mathrm{WK}, i}^{\mathcal{F}}$ and the outfluxes $Q_{\mathrm{Out}, i}^{\mathcal{F}}$ through the different outflow boundaries $\Gamma_{\text {Out }, i}^{\mathcal{F}}$ can be derived [77]. Hence, the interface traction on the fluid (see equation (5)) can be expressed directly by an integrated quantity of the velocity unknowns. No additional field for the windkessel subproblems and no additional unknowns for the windkessel pressure are introduced.

The linear system (60) is solved by a parallel preconditioned GMRES [85] with FSI specific block preconditioning based on algebraic multigrid [36].

\subsection{Monolithic SSI}

The (fluid-scalar)-(structure-scalar) interaction problem is solved by a monolithic approach too. The monolithic linear problem to solve for the incremental concentrations in the fluid $\Delta \mathbf{c}_{h}^{\mathcal{F S}}$ and in the structure $\Delta \mathbf{c}_{h}^{\mathcal{S S}}$ reads [108]

$$
\begin{aligned}
& \left(\begin{array}{ll}
\frac{\partial}{\partial \mathbf{c}_{h}^{\mathcal{F}}} \mathbf{r}^{\mathcal{F S}} & \frac{\partial}{\partial \mathbf{c}_{h \mathcal{S}}^{\mathcal{S}}} \mathbf{r}^{\mathcal{F S}} \\
\frac{\partial}{\partial \mathbf{c}_{h}^{\mathcal{F}}} \mathbf{r}^{\mathcal{S} \mathcal{S}} & \frac{\partial}{\partial \mathbf{c}_{h}^{\mathcal{S} S}} \mathbf{r}^{\mathcal{S} \mathcal{S}}
\end{array}\right)_{i}^{n+1}\left(\begin{array}{c}
\Delta \mathbf{c}_{h}^{\mathcal{F} \mathcal{S}} \\
\Delta \mathbf{c}_{h}^{\mathcal{S} \mathcal{S}}
\end{array}\right)_{i+1}^{n+1} \\
& =-\left(\begin{array}{c}
\mathbf{r}^{\mathcal{F S}} \\
\mathbf{r}^{\mathcal{S S}}
\end{array}\right)_{i}^{n+1}
\end{aligned}
$$

where $n=0,1, \ldots, n_{T}$ is the time step and $i=0,1, \ldots$ is the Newton step. $\mathbf{r}^{\mathcal{F} \mathcal{S}}$ and $\mathbf{r}^{\mathcal{S}}$ denote the fully discrete fluid-scalar and structure-scalars residuals, respectively. The Newton iteration for the time step $n$ is stopped if both 2-norms $\left\|\mathbf{r}^{\mathcal{F} \mathcal{S}}\right\|_{2}$ and $\left\|\mathbf{r}^{\mathcal{S S}}\right\|_{2}$ scaled to their lengths are below $10^{-5}$.

Solving of the linear system (61) for the unknown step increments $\Delta \mathbf{c}_{h}^{\mathcal{F}}$ and $\Delta \mathbf{c}_{h}^{\mathcal{S S}}$ is again performed by parallel GMRES [85] with block preconditioning [36].

\subsection{Meshing}

Our discretizations are generated using Trelis (Csimsoft) and satisfy the following properties:

- It is conforming on the FS3I interface $\Gamma_{\text {FS3I }}$.

- All elements are characterized by a characteristic element length $h$.

- In the fluid domain $\Omega^{\mathcal{F}}$ a boundary refinement is introduced to better resolve velocity gradients and concentrations $c_{\mathrm{LDL}}$ near the FS3I interface $\Gamma_{\mathrm{FS} 3 \mathrm{I}}^{\mathcal{F}}$.
- The structure domain $\Omega^{\mathcal{S}}$ is meshed using hexahedral elements with F-bar technology [90,25].

The lumen of patient-specific geometries of the aortic arch are non-trivial to mesh with hexahedral elements. Hence, a tetrahedral dominated mesh with a hexahedral boundary layer is generated. The tetrahedral and hexahedral elements of the fluid mesh are connected by pyramid shaped elements. The boundary layer consists of 4 layers of hexahedral elements with individual layer thicknesses of $\frac{h}{2}, \frac{h}{4}, \frac{h}{8}$ and $\frac{h}{16}$ towards the direction of the FS3I interface $\Gamma_{\text {FS3I }}$. The structure domain is adjacent to the FS3I interface $\Gamma_{\text {FS3I }}^{\mathcal{F}}$ with thickness $T$. It is meshed in 6 element layers with an equidistant thickness. The meshes of the ALE domain $\Omega^{\mathcal{G}}$ and the fluid-scalar domain $\Omega^{\mathcal{F S}}$ equal the fluid mesh. The mesh of the structure-scalars domain $\Omega^{\mathcal{S S}}$ is equal to the structure mesh.

\section{Computational case study and results}

In the following section we calibrate the presented mathematical and computational model to a murine-specific case, give the computational results of the case study and compare the results with various literature. The geometry of the case study is a murine-specific reconstruction of the lumen of a non-atherosclerotic mouse (type C57BL/6J), see Figure 4. It was segmented from an in vivo magnetic resonance angiography by our medical partners. The segmentation was performed in a semi-automatic fashion using Mimics (Materialise). The measurement was performed on a horizontal bore $7 \mathrm{~T}$ small animal scanner (Discovery MR901, GE Healthcare) applying a ECG-triggered 3D gradient echo sequence achieving an in-plane resolution of $59 \mu \mathrm{m}$ with a slice thickness of $250 \mu \mathrm{m}$. The achieved resolution did not allow for an exact segmentation of the artery wall. As only a small part of the artery tree is considered, the variation of the wall thickness is neglected and a constant wall thickness $T$ is employed.

The discretization for the performed case study of our murine-specific geometry is visualized in Figure 4. A detailed summary of the utilized mesh as described in section 3.6 with a characteristic element length $h=0.06 \mathrm{~mm}$ (for comparison: the radius of the inflow boundary $\Gamma_{\text {In }}^{\mathcal{F}}$ is $R_{\mathrm{In}}=0.57 \mathrm{~mm}$ ) and a constant artery wall thickness $T=0.08 \mathrm{~mm}$ [104] is given in Table 1. A mesh convergence analysis is performed in section 4.3 . 


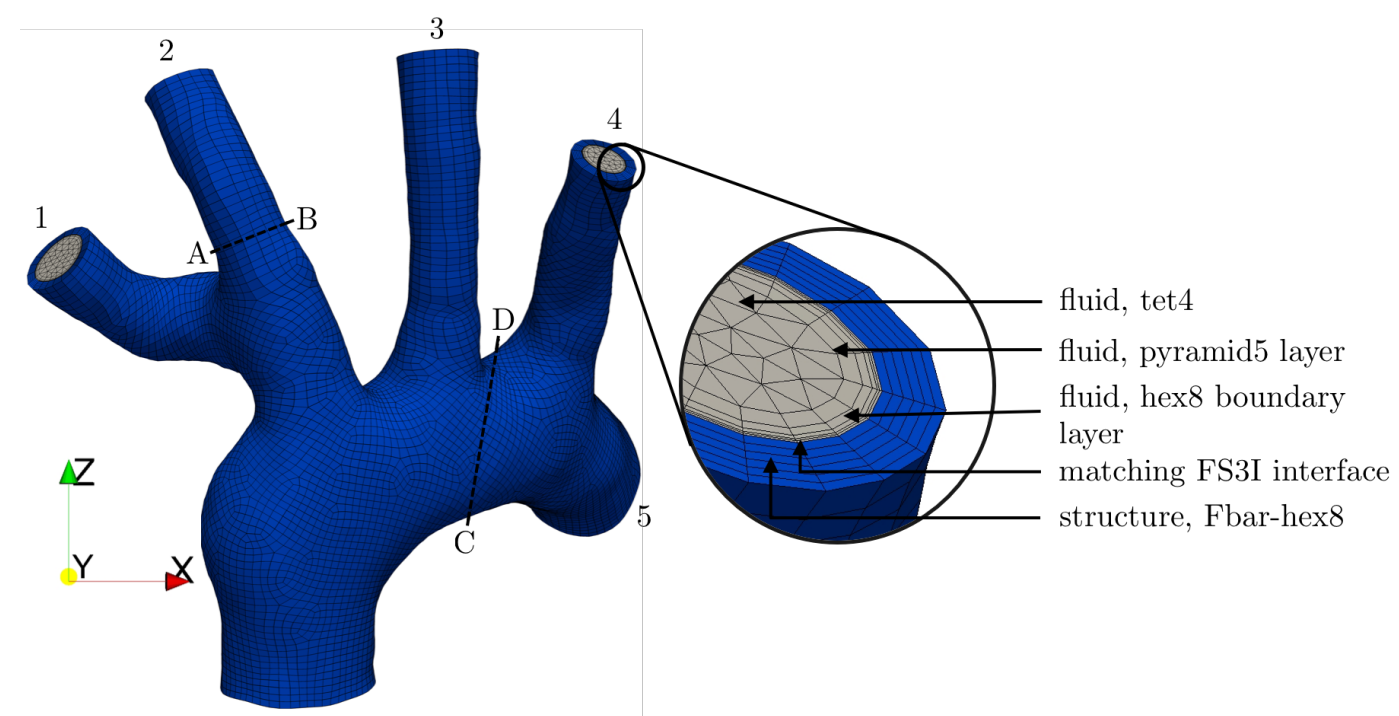

Fig. 4 Conforming FS3I mesh generated using Trelis consisting of tetrahedral, hexahedral and pyramid shaped elements with a characteristic element length $h=0.06 \mathrm{~mm}$. Gray represents the mesh used for the fluid domain $\Omega^{\mathcal{F}}$, ALE domain $\Omega^{\mathcal{G}}$ and the fluid-scalar domain $\Omega^{\mathcal{F} \mathcal{S}}$. Blue represents the mesh used for the structure domain $\Omega^{\mathcal{S}}$ and the structure-scalars domain $\Omega^{\mathcal{S} \mathcal{S}}$. The numbers indicate the numbering of the outlet boundaries and the lines AB and CD are the profile lines used in the mesh convergence analysis.

Table 1 Overview of the number of elements, number of nodes, degrees of freedom (DOF) per node and total DOF of the fluid domain $\Omega^{\mathcal{F}}$, structure domain $\Omega^{\mathcal{S}}$, ALE domain $\Omega^{\mathcal{G}}$, fluid-scalar domain $\Omega^{\mathcal{F} \mathcal{S}}$ structure-scalars domain $\Omega^{\mathcal{S} \mathcal{S}}$ and combinations of the mesh with a characteristic element length $h=0.06 \mathrm{~mm}$.

\begin{tabular}{c|c|c|c|c} 
Domains & \# Elements & \# Nodes & \# DOF per node & \# DOF \\
\hline$\Omega^{\mathcal{F}}$ & Tet4 315896, Pyr5 9523, Hex8 47615 & 108986 & 4 & 435944 \\
$\Omega^{\mathcal{S}}$ & Hex8 57138 & 67298 & 3 & 201894 \\
$\Omega^{\mathcal{G}}$ & Tet4 315896, Pyr5 9523, Hex8 47615 & 108986 & 3 & 326958 \\
$\Omega^{\mathcal{S} S}$ & Tet4 315896, Pyr5 9523, Hex8 47615 & 108986 & 1 & 108986 \\
$\Omega^{\mathcal{S}}$ & Hex8 57138 & 67298 & 2 & 134596 \\
\hline \hline$\Omega^{\mathcal{F}} \cup \Omega^{\mathcal{S}} \cup \Omega^{\mathcal{G}}$ & 430172 & 176284 & 5.47 & 964796 \\
$\Omega^{\mathcal{F S} \cup \Omega^{\mathcal{S}}}$ & 430172 & 176284 & 1.38 & 243582 \\
\hline \hline All & 430172 & 176284 & 6.85 & 1208378
\end{tabular}

\subsection{Model parameters}

Due to a lack of suitable in vivo data, we use an exemplary set of key physiological data of mice from literature and derive from it a set of model parameters for the given patient-specific geometry. The experimental results in [52] are utilized providing a complete set of physiological data - the mean volume influx $\bar{Q}_{\text {In }}^{\mathcal{F}}$, the length of the cardiac cycle $T_{\mathrm{Cycl}}$, the diastolic pressure $p_{\text {dia }}{ }^{\mathcal{F}}$ and the systolic pressure $p_{\text {sys }}{ }^{\mathcal{F}}$ - from a single source. However, this data-set is just one possible choice representing the mice studied in [52] where the systolic pressure $p_{\text {sys }} \mathcal{F}$ seems to be low compared to other studies $[103,4]$. First, we give an overview of the parameters which have to be calibrated to the specific geometry and afterwards list the remaining parameters taken from literature, see Tables 3 and 4 .
For the prescribed inflow velocities on $\Gamma_{\text {In }}^{\mathcal{F}}$ given by equation (4) the velocity profile $g(t, \mathbf{x})$ and the total volume influx $Q_{\operatorname{In}}^{\mathcal{F}}(t)$ need to be specified. For the inflow profile $g(t, \mathbf{x})$ a $9^{\text {th }}$ order polynomial-shaped function is utilized [88], which is superimposed by a womersley profile respecting the influence of the oscillatory influx on the velocity profile [49]. The temporal shape of the volume influx $Q_{\text {In }}^{\mathcal{F}}(t)$ is taken from [77] and is scaled such that the length of the cardiac cycle $T_{\mathrm{Cycl}}$ and the mean influx $\bar{Q}_{\text {In }}^{\mathcal{F}}$ fits to the murine physiology $[52,29,4]$ : $T_{\text {Cycl }}=0.1 \mathrm{~s}, \bar{Q}_{\text {In }}^{\mathcal{F}}=16.2 \frac{\mathrm{ml}}{\mathrm{min}}$. The resulting prescribed influx $Q_{\operatorname{In}}^{\mathcal{F}}(t)$ is plotted in Figure 5.

The parameters of each of the five three-element Windkessels must be fitted to the murine physiology and the specific geometry. For the murine physiology a diastolic pressure $p_{\text {dia }}^{\mathcal{F}}=77 \mathrm{mmHg}=10265.8 \mathrm{~Pa}$ and a systolic pressure $p_{\text {sys }}^{\mathcal{F}}=100 \mathrm{mmHg}=13332.2 \mathrm{~Pa}$ are assumed as measured in [52]. 


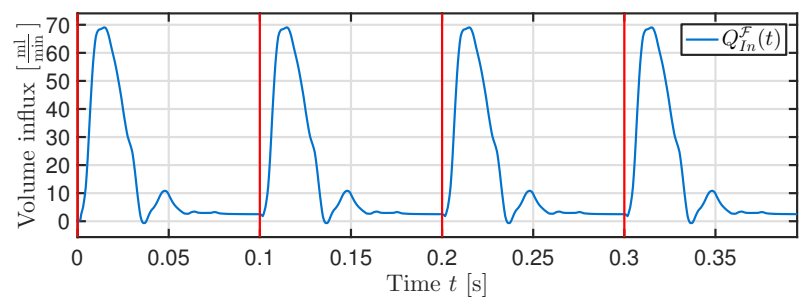

Fig. 5 Prescribed volume influx $Q_{\text {In }}^{\mathcal{F}}(t)$ through $\Gamma_{\text {In }}^{\mathcal{F}}$. The function is periodic with a periodicity of $T_{\mathrm{Cycl}}=0.1 \mathrm{~s}$.

The compliance of the geometry is approximated by a simulation of the structure subproblem, where only a hydrostatic pressure is applied. Thereby, the geometry is first prestressed to the diastolic pressure $p_{\text {dia }}$ and afterwards the pressure is further increased to the systolic pressure $p_{\text {sys }}^{\mathcal{F}}$. Then the compliance of the given geometry can be approximated by

$C_{\text {geo }} \approx \frac{V_{\text {sys }}^{\mathcal{F}}-V_{\text {dia }}^{\mathcal{F}}}{p_{\text {sys }}^{\mathcal{F}}-p_{\text {dia }}^{\mathcal{F}}}=11.86 \cdot 10^{-4} \frac{\mathrm{mm}^{3}}{\mathrm{~Pa}}$,

where $V_{\text {sys }}^{\mathcal{F}}$ and $V_{\text {dia }}^{\mathcal{F}}$ are the volume of the lumen measured at the systolic and diastolic pressure level, respectively. Furthermore, the approximated portions of volume outflux $\% Q_{\text {Out }, i}^{\mathcal{F}}$ through each of the five outflow boundaries $\Gamma_{\text {Out }, i}^{\mathcal{F}}$ is approximated by the ratio of the surface area of $\Gamma_{\text {Out }, i}^{\mathcal{F}}$ to the total surface area of all five outflow boundaries. Following the approach of [105] and [50], we achieve Windkessel parameters as given in Table 2.

Table 2 Parameters for the calibration of the three-element Windkessel and calibrated results. Approximated portion of volume outflux $\% Q_{\mathrm{Out}, i}^{\mathcal{F}}$ and resulting characteristic resistance $R_{\mathrm{C}, i}$, peripheral resistance $R_{\mathrm{P}, i}$ and artery compliance $C_{i}$ of each of the five three-element Windkessels. All units are in $\mathrm{mm}, \mathrm{g}, \mathrm{s}$.

\begin{tabular}{|c|c|c|c|c|}
\hline$i$ & $\% Q_{\text {Out }, i}^{\mathcal{F}}$ & $R_{\mathrm{C}, i}$ & $R_{\mathrm{P}, i}$ & $C_{i} \cdot 10^{4}$ \\
\hline 1 & $11.36 \%$ & $16.93 \frac{\mathrm{Pa} \mathrm{s}}{\mathrm{mm}^{3}}$ & $384.18 \frac{\mathrm{Pa} \mathrm{s}}{\mathrm{mm}^{3}}$ & $4.29 \frac{\mathrm{mm}^{3}}{\mathrm{~Pa}}$ \\
\hline 2 & $8.97 \%$ & $19.56 \frac{\mathrm{Pa} \mathrm{s}}{\mathrm{mm}^{3}}$ & $426.39 \frac{\mathrm{Pa} \mathrm{s}}{\mathrm{mm}^{3}}$ & $3.29 \frac{\mathrm{mm}^{3}}{\mathrm{~Pa}}$ \\
\hline 3 & $12.73 \%$ & $12.00 \frac{\mathrm{Pa} \mathrm{s}^{3}}{\mathrm{~mm}^{3}}$ & $369.63 \frac{\mathrm{Pa} \mathrm{s}^{3}}{\mathrm{~mm}^{3}}$ & $4.81 \frac{\mathrm{mm}^{3}}{\mathrm{~Pa}}$ \\
\hline 4 & $7.55 \%$ & $21.49 \frac{\mathrm{Pa} \mathrm{s}^{3}}{\mathrm{~mm}^{3}}$ & $441.62 \frac{\mathrm{Pa}}{\mathrm{mm}^{3}}$ & $2.86 \frac{\mathrm{mm}^{3}}{\mathrm{~Pa}}$ \\
\hline 5 & $59.39 \%$ & $4.67 \frac{\mathrm{Pa} \mathrm{s}}{\mathrm{mm}^{3}}$ & $69.73 \frac{\mathrm{Pa} \mathrm{s}^{3}}{\mathrm{~mm}^{3}}$ & $21.50 \frac{\mathrm{mm}^{3}}{\mathrm{~Pa}}$ \\
\hline
\end{tabular}

The growth parameter $\alpha$ in equation (18) corresponds to the amount of unite volume occupied by a unit scalar of foam cells. We follow the approach of [34] and ask for a constant mass density $\varrho^{\mathcal{S}}$ of the artery wall, which in later states consists of both, the healthy aortic tissue and the foam cell population. Hence, we utilize:

$\alpha=\frac{1}{\varrho_{0}^{\mathcal{S}}}=1.0 \cdot 10^{3} \frac{\mathrm{mm}^{3}}{\mathrm{~g}}$.

The remaining parameters of the FSI subproblem are independent of the specific geometry and are taken from literature. However, no complete data-set based on murine experiments exists such that the used parameters for the Carreau-Yasuda model, the surrounding tissue and the constitutive laws are based on human experiments. An overview of fitted as well as the remaining parameters of the FSI subproblem is found in Table 3 .

For the calibration of the permeability scaling factor $\left.s\left(\|<\boldsymbol{\tau}^{\mathcal{F}}\right\rangle \|\right)$ in equation (45) we generalize the idea of [11]. We determine the two model parameters $m_{1}$ and $m_{2}$ of the monotonically decreasing function such that the following two conditions are fulfilled:

1. The scaling factor vanishes, when the norm $\left\|\boldsymbol{\tau}^{\mathcal{F}}\right\|$ of the wall-shear stresses is equal to the reference value $\left\|\bar{\tau}^{\mathcal{F}}\right\|$. This reference value is approximated by considering a stationary Poiseuille flow with an equivalent total volume influx $\bar{Q}_{\text {In }}^{\mathcal{F}}$ through a straight pipe with the same radius $R_{\text {In }}$ as the inflow boundary $\Gamma_{\text {In }}^{\mathcal{F}}$. Hence, we require

$$
\begin{aligned}
s\left(\left\|\overline{\boldsymbol{\tau}}^{\mathcal{F}}\right\|\right) & =1 \text { with } \\
\left\|\overline{\boldsymbol{\tau}}^{\mathcal{F}}\right\| & =\frac{4}{\pi} \frac{\eta_{\infty} \bar{Q}_{\mathrm{In}}^{\mathcal{F}}}{R_{\mathrm{In}}^{3}}=6.404 \mathrm{~Pa} .
\end{aligned}
$$

2. According to measurements in [42] the local permeability of the endothelium with respect to LDL in regions with high permeability is up to a factor of 25 higher than in regions with low permeability. We assume the harmonic mean of those two extrema being the case of $s\left(\left\|\bar{\tau}^{\mathcal{F}}\right\|\right)=1$. Since the permeability is highest for the case $\left\|\boldsymbol{\tau}^{\mathcal{F}}\right\|=0$ we require the PSF to fulfill

$s(0)=5$.

The two assumptions lead to

$$
\begin{aligned}
s\left(\left\|<\boldsymbol{\tau}^{\mathcal{F}}>\right\|\right)=\frac{1}{\ln (2)} \ln \left(1+\frac{\left(1+\frac{1}{2^{s(0)}-2}\right)\left\|\overline{\boldsymbol{\tau}}^{\mathcal{F}}\right\|}{\left\|<\boldsymbol{\tau}^{\mathcal{F}}>\right\|+\frac{1}{2^{s(0)}-2}\left\|\overline{\boldsymbol{\tau}}^{\mathcal{F}}\right\|}\right) \\
=\frac{1}{\ln (2)} \ln \left(1+\frac{6.618 \mathrm{~Pa}}{\left\|<\boldsymbol{\tau}^{\mathcal{F}}>\right\|+0.213 \mathrm{~Pa}}\right),
\end{aligned}
$$

which is plotted in Figure 6 .

An overview of the calibrated and remaining physiological parameters for the SSI subproblem is found in Table 4. 
Table 3 Overview of all parameters of the fluid-structure interaction subproblem. Parameters are sorted by the first appearance in the model. All units are in $\mathrm{mm}, \mathrm{g}, \mathrm{s}, \mathrm{Pa}$.

\begin{tabular}{|c|c|c|c|c|}
\hline Parameter & Equation & Description & Value & Source \\
\hline$\varrho^{\mathcal{F}}$ & $(1)$ & Mass density of blood & $1.05 \cdot 10^{-3} \frac{\mathrm{g}}{\mathrm{mm}^{3}}$ & {$[51]$} \\
\hline$\eta_{\infty}$ & (3) & Dynamic viscosity of blood for high shear rates & $3.45 \cdot 10^{-3} \mathrm{~Pa} \mathrm{~s}$ & [16] \\
\hline$\eta_{0}$ & (3) & Dynamic viscosity of blood for zero shear rates & $5.6 \cdot 10^{-2} \mathrm{~Pa} \mathrm{~s}$ & {$[16]$} \\
\hline$\kappa$ & (3) & Characteristic time of Curreau-Yasuda model & $1.902 \mathrm{~s}$ & {$[16]$} \\
\hline$b$ & (3) & Parameter of Curreau-Yasuda model & 1.25 & [16] \\
\hline$a$ & (3) & Parameter of Curreau-Yasuda model & 0.624 & [16] \\
\hline$Q_{\text {In }}^{\mathcal{F}}(t)$ & $(4)$ & Influx rate over time & Figure 5 & {$[77,52]$} \\
\hline$T_{\mathrm{Cycl}}$ & (4) & Length of cardiac cycle & $0.1 \mathrm{~s}$ & {$[29]$} \\
\hline $\bar{Q}_{\text {In }}^{\mathcal{F}}$ & (4) & Mean influx rate & $270.0 \frac{\mathrm{mm}^{3}}{\mathrm{~s}}$ & {$[52]$} \\
\hline$C_{i}$ & (6) & Artery compliances of Windkessels on $\Gamma_{\text {Out }, i}^{\mathcal{F}}$ & Table 2 & Table 2 \\
\hline$R_{\mathrm{C}, i}$ & (6) & Characteristic resistances of Windkessels on $\Gamma_{\text {Out }, i}^{\mathcal{F}}$ & Table 2 & Table 2 \\
\hline$R_{\mathrm{P}, i}$ & (6) & Peripheral resistances of Windkessels on $\Gamma_{\mathrm{Out}, i}^{\mathcal{F}}$ & Table 2 & Table 2 \\
\hline$\varrho_{0}^{\mathcal{S}}$ & $(7)$ & Reference mass density of artery wall & $1.0 \cdot 10^{-3} \frac{\mathrm{g}}{\mathrm{mm}^{3}}$ & {$[34]$} \\
\hline$k_{\text {Wall }}^{\mathcal{S}}$ & $(8)$ & Spring stiffness of embedded tissue & $5.0 \cdot 10^{3} \frac{\mathrm{Pa}^{\circ}}{\mathrm{mm}}$ & {$[22,71]$} \\
\hline$c_{\text {Wall }}^{\mathcal{S}}$ & (8) & Dashpot viscosity of embedded tissue & $100.0 \frac{\mathrm{Pas}}{\mathrm{mm}}$ & {$[71]$} \\
\hline$k_{\text {Out }}^{\mathcal{S}}$ & (9) & Spring stiffness of succeeding tissue & $2.0 \cdot 10^{4} \frac{\mathrm{ma}}{\mathrm{mm}}$ & [71] \\
\hline$c_{\text {Out }}^{\mathcal{S}}$ & $(9)$ & Dashpot viscosity of succeeding tissue & $100.0 \frac{\mathrm{Pas}}{\mathrm{mm}}$ & {$[71]$} \\
\hline$\alpha$ & $(18),(22)$ & Growth parameter & $1.0 \cdot 10^{3} \frac{\mathrm{mm}^{3}}{\mathrm{~g}}$ & eq. (63) \\
\hline$c_{0, \text { Ao }}$ & $(23)$ & Stiffness of aortic ground material & $47.43 \cdot 10^{3} \mathrm{~Pa}$ & [83] \\
\hline$c_{1,1}$ & $(23)$ & Stiffness of first fiber & $35.23 \cdot 10^{3} \mathrm{~Pa}$ & {$[83]$} \\
\hline$c_{2,1}$ & $(23)$ & Exponential parameter of first fiber & $7.65 \cdot 10^{-6}$ & [83] \\
\hline$\delta_{1}$ & $(23)$ & Angular of first fiber & $0^{\circ}$ & {$[83]$} \\
\hline$c_{1,2}$ & $(23)$ & Stiffness of second fiber & $40.84 \cdot 10^{3} \mathrm{~Pa}$ & [83] \\
\hline$c_{2,2}$ & (23) & Exponential parameter of second fiber & 0.1 & [83] \\
\hline$\delta_{2}$ & (23) & Angular of second fiber & $90^{\circ}$ & [83] \\
\hline$c_{1,3}$ & $(23)$ & Stiffness of third fiber & $15.21 \cdot 10^{3} \mathrm{~Pa}$ & [83] \\
\hline$c_{2,3}$ & $(23)$ & Exponential parameter of third fiber & 2.58 & {$[83]$} \\
\hline$\delta_{3}$ & $(23)$ & Angular of third fiber & $48.98^{\circ}$ & {$[83]$} \\
\hline$c_{1,4}$ & (23) & Stiffness of fourth fiber & $15.21 \cdot 10^{3} \mathrm{~Pa}$ & [83] \\
\hline$c_{2,4}$ & $(23)$ & Exponential parameter of fourth fiber & 2.58 & [83] \\
\hline$\delta_{4}$ & $(23)$ & Angular of fourth fiber & $-48.98^{\circ}$ & {$[83]$} \\
\hline$\tau_{\mathrm{FC}}$ & $(24)$ & Relaxation time of lipid material & $47.5 \cdot 10^{-3} \mathrm{~s}$ & {$[72]$} \\
\hline$c_{0, \mathrm{FC}}$ & $(24)$ & Stiffness of lipid material & $10.0 \cdot 10^{3} \mathrm{~Pa}$ & {$[5]$} \\
\hline
\end{tabular}

Table 4 Overview of all parameters of the SSI subproblem. Parameters are sorted by the first appearance in the model. All units are in $\mathrm{mm}, \mathrm{g}, \mathrm{s}, \mathrm{Pa}$.

\begin{tabular}{c|c|c|c|c} 
Parameter & Equation & Description & Value & Source \\
\hline$D_{\mathrm{LD}}^{\mathcal{F}}$ & $(27)$ & Diffusivity of LDL in the blood & $3.07 \cdot 10^{-5} \frac{\mathrm{mm}^{2}}{\mathrm{~s}}$ & {$[64,98]$} \\
$c_{\mathrm{LDL}, \text { In }}^{\mathcal{S} D}$ & $(28)$ & Concentration of LDL at $\Gamma_{\mathrm{In}}^{\mathcal{F} \mathcal{S}}$ & $1.22 \cdot 10^{-6} \frac{\mathrm{g}}{\mathrm{mm}^{3}}$ & {$[33]$} \\
$D_{\mathrm{L} \mathcal{S}}^{\mathcal{S}}$ & $(32)$ & Diffusivity of LDL in the artery wall & $5.4 \cdot 10^{-8} \frac{\mathrm{mm}^{2}}{\mathrm{~s}}$ & {$[98,42]$} \\
$D_{\mathrm{FC}}^{\mathcal{S}}$ & $(32)$ & Diffusivity of foam cells in the artery wall & 0.0 & {$[34]$} \\
$P_{D}$ & $(43)$ & Diffusive permeability of the endothelium & $1.7 \cdot 10^{-8} \frac{\mathrm{mm}}{\mathrm{s}}$ & {$[97,106]$} \\
$m_{1}$ & $(45)$ & Permeability scaling factor parameter & $6.618 \mathrm{~Pa}$ & eq. (67) \\
$m_{2}$ & $(45)$ & Permeability scaling factor parameter & $0.213 \mathrm{~Pa}$ & eq. (67)
\end{tabular}

\subsection{Dimensionless parameters}

From the parameters in Table 3 and Table 4 dimensionless parameters are calculated. For the fluid problem the Reynolds number $R e$ at the inflow boundary is given by

$R e=\frac{2 \varrho^{\mathcal{F}}\left\|\mathbf{u}^{\mathcal{F}}\right\| R_{\text {In }}}{\eta_{\infty}}$

where $\mathbf{u}^{\mathcal{F}}$ is the characteristic velocity. Using the peak velocity at the inflow boundary $\Gamma_{\text {In }}^{\mathcal{F}}$, i.e. $\max _{\mathbf{u}^{\mathcal{F}} \in \Gamma_{\text {In }}^{\mathcal{F}}}\left\|\mathbf{u}^{\mathcal{F}}\right\|$ results in an approximation for the peak Reynolds number $R e_{\text {Peak }}=411.3$. Using the temporal and spatial mean of the velocities on the inflow boundary results in an approximation for the mean Reynolds number $R e_{\text {Mean }}=91.9$. The peak and mean Reynolds numbers are slightly bigger than found in literature $[29,4$, 48,94] The Womersley number Wo of the fluid is given 


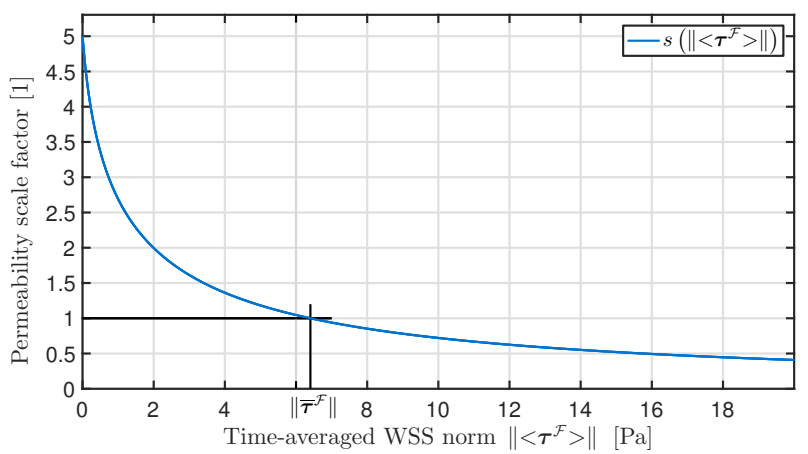

Fig. 6 Calibrated law for the permeability scaling factor $\left.s\left(\|<\boldsymbol{\tau}^{\mathcal{F}}\right\rangle \|\right)$ plotted over the norm of the time-averaged wallshear stresses $\left\|<\tau^{\mathcal{F}}>\right\|$ of the fluid. $\left\|\bar{\tau}^{\mathcal{F}}\right\|=6.404 \mathrm{~Pa}$ is the approximated reference value of the WSS.

by

$W o=\sqrt{\frac{2 \pi \varrho^{\mathcal{F}} R_{\mathrm{In}}^{2}}{T_{\mathrm{Cycl}} \eta_{\infty}}}=2.49$,

fitting very well to the murine physiology [29,4,48,94]. Since both Reynolds numbers $R e_{\text {Peak }}$ and $R e_{\text {Mean }}$ as well as the Womersley number $W o$ are small, the behavior of the fluid is viscous-dominated and in the laminar regime.

The dimensionless parameter for the transportation of LDL with the blood flow governed by the advectiondiffusion equation is the Peclet number $P e$ given by

$P e=\frac{2\left\|\mathbf{u}^{\mathcal{F}}\right\| R_{\mathrm{In}}}{D_{\mathrm{LDL}}^{\mathcal{F} \mathcal{S}}}$.

Inserting again the peak and mean velocity the range for the Peclet number is $P e \in\left[9.82 \cdot 10^{6} ; 4.40 \cdot 10^{7}\right]$ being in the physiological regime of LDL transport [91]. Hence, the transportation with the blood flow is advection dominated, except for regions close to the no-slip fluidstructure interface $\Gamma_{\text {FS3I }}^{\mathcal{F}}$.

\subsection{Convergence analysis}

To prove the validity of the computational results a spatial and temporal convergence analysis is performed. We exemplarily check the convergence of the fluid velocities $\mathbf{u}^{\mathcal{F}}$ and the ALE displacements $\mathbf{d}^{\mathcal{G}}$ over two distinct lines $\mathrm{AB}$ and $\mathrm{CD}$ as indicated in Figure 4.

The spatial convergence is analyzed by utilizing the constant time step size $\Delta t=2.5 \cdot 10^{-4} \mathrm{~s}$ in combination with meshes as described in section 3.6 with characteristic element lengths $h$ of $0.04 \mathrm{~mm}, 0.06 \mathrm{~mm}$, $0.09 \mathrm{~mm}$ and $0.12 \mathrm{~mm}$. The velocity profiles of the four meshes over the lines $\mathrm{AB}$ and $\mathrm{CD}$ at the peak of the systolic phase of the fifth cardiac cycle (i.e. $t=$
$0.412 \mathrm{~s}$ ) is plotted in Figure 7. The meshes with $h=$ $0.04 \mathrm{~mm}$ and $h=0.06 \mathrm{~mm}$ posses qualitatively the same behavior. Quantitatively, the relative $L_{2}$-error of the velocities $\frac{\left\|\mathbf{u}_{h=0.04 \mathrm{~mm}}^{\mathcal{F}}-\mathbf{u}_{h=0.06 \mathrm{~mm}}^{\mathcal{F}}\right\|}{\left\|\mathbf{u}_{h=0.06 \mathrm{~mm}}^{\mathcal{F}}\right\|}$ and the displacements $\frac{\left\|\mathbf{d}_{h=0.04 \mathrm{~mm}}^{\mathcal{G}}-\mathbf{d}_{h=0.06 \mathrm{~mm}}^{\mathcal{G}}\right\|}{\left\|\mathbf{d}_{h=0.06 \mathrm{~mm}}^{\mathcal{G}}\right\|}$ over the lines AB and CD between the finest two meshes are below 1,6\%.

Additionally to the velocities and displacements, we exemplarily check the spatial convergence of the WSS and von Mises stresses at the four intersection points of the fluid-structure interface $\Gamma_{\text {FS3I }}^{\mathcal{F}}$ and the lines AB and CD. The relative errors of the WSS between the finest two meshes at these four points are below $2.75 \%$. The relative errors of the von Mises stresses between the finest two meshes at these four points are below $7.38 \%$ but are well within the asymptotic range.

To analyze the temporal convergence the mesh with a characteristic element length $h=0.06 \mathrm{~mm}$ is utilized with three time step sizes $\Delta t$ of $1.25 \cdot 10^{-4} \mathrm{~s}, 2.5 \cdot 10^{-4} \mathrm{~s}$ and $5.0 \cdot 10^{-4} \mathrm{~s}$. Again the velocity profiles over the lines $\mathrm{AB}$ and $\mathrm{CD}$ are analyzed and plotted at time $t=$ $0.412 \mathrm{~s}$ in Figure 8. Temporal convergence is sufficiently reached with a time step size of $\Delta t=2.5 \cdot 10^{-4} \mathrm{~s}$. Quantitatively, the relative $L_{2}$-error of the velocities and the displacements over the lines $\mathrm{AB}$ and $\mathrm{CD}$ between the smallest two time step sizes are below $1,3 \%$.

The qualitatively same spatial and temporal convergence behavior was observed for other points and profile lines too. As the wall stress is no quantity of particular interest in the present model, we further employ the mesh with a characteristic element length $h=0.06 \mathrm{~mm}$ in combination with a constant time step size $\Delta t=2.5 \cdot 10^{-4} \mathrm{~s}$.

\subsection{Model validation}

Due to lack of detailed experimental data, the presented mathematical model and its parameters are solely qualitatively validated by comparing the computational results to cardiovascular measurements and computational results from literature. The Windkessel subproblems determining the pressure of the fluid starts with an unphysiological zero pressure and requires approximately four cardiac cycles to reach a periodic state. Hence, in the following the computed results from the seventh simulated cardiac cycle are utilized. Exemplarily, the Windkessel pressure at the outflow boundary $\Gamma_{\text {Out }, 5}$ over time $t$ is plotted in Figure 9. In its periodic state the Windkessel subproblem at $\Gamma_{\text {Out, } 5}$ is oscillating between the diastolic pressure $p_{\text {dia }} \approx 74.0 \mathrm{mmHg}=9867.0 \mathrm{~Pa}$ and systolic pressure $p_{\text {sys }} \approx 102.5 \mathrm{mmHg}=13660.6 \mathrm{~Pa}$, being close to the assumed pressure levels in [52]. Still, the systolic pressure is approximately $10-20 \%$ low compared to 


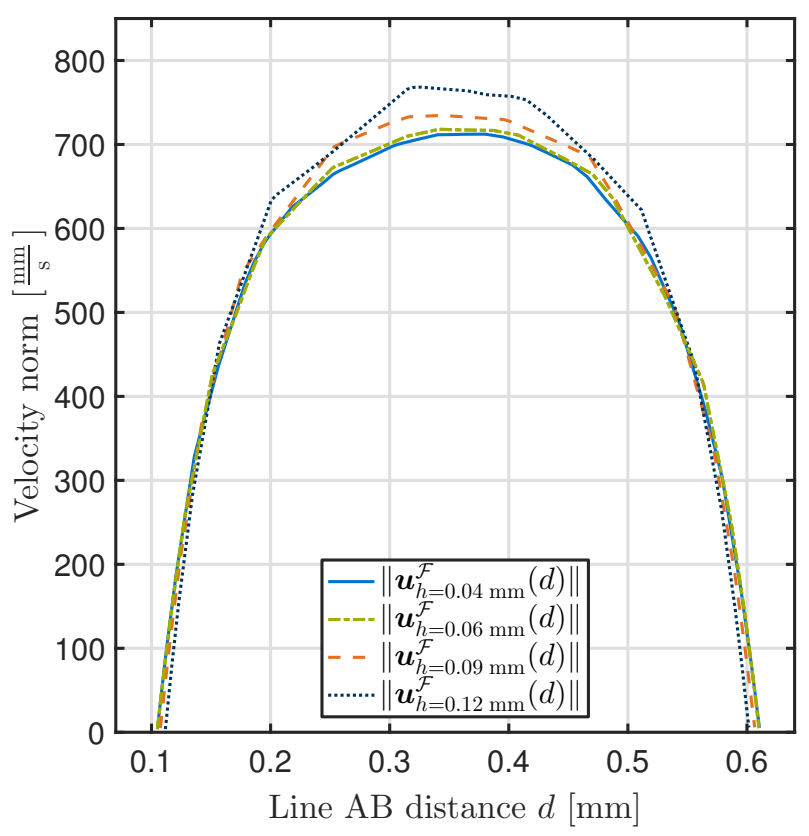

(a) Comparison of the velocitiy profiles over the line $\mathrm{AB}$ (see Figure 4) for different meshes with characteristic element lengths $h$ of $0.04 \mathrm{~mm}, 0.06 \mathrm{~mm}, 0.09 \mathrm{~mm}, 0.12 \mathrm{~mm}$ and constant time step size $\Delta t=2.5 \cdot 10^{-4} \mathrm{~s}$ at time $t=0.412 \mathrm{~s}$.

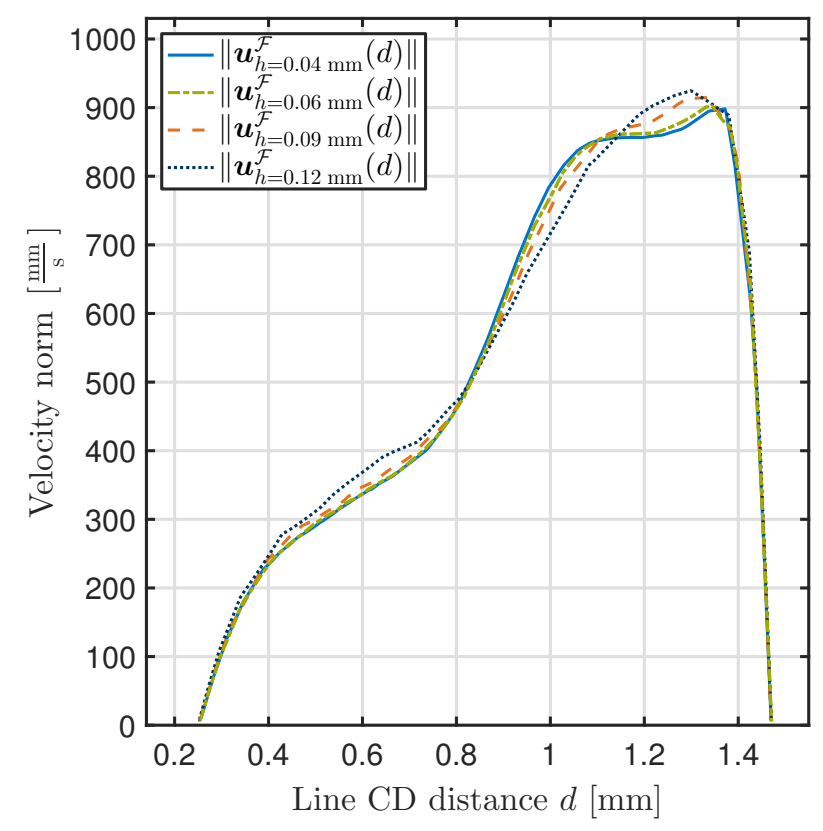

(b) Comparison of the velocitiy profiles over the line CD (see Figure 4) for different meshes with characteristic element lengths $h$ of $0.04 \mathrm{~mm}, 0.06 \mathrm{~mm}, 0.09 \mathrm{~mm}, 0.12 \mathrm{~mm}$ and constant time step size $\Delta t=2.5 \cdot 10^{-4} \mathrm{~s}$ at time $t=0.412 \mathrm{~s}$.

Fig. 7 Spatial convergence study.

other literature, see e.g. [103,4]. However, the quantities

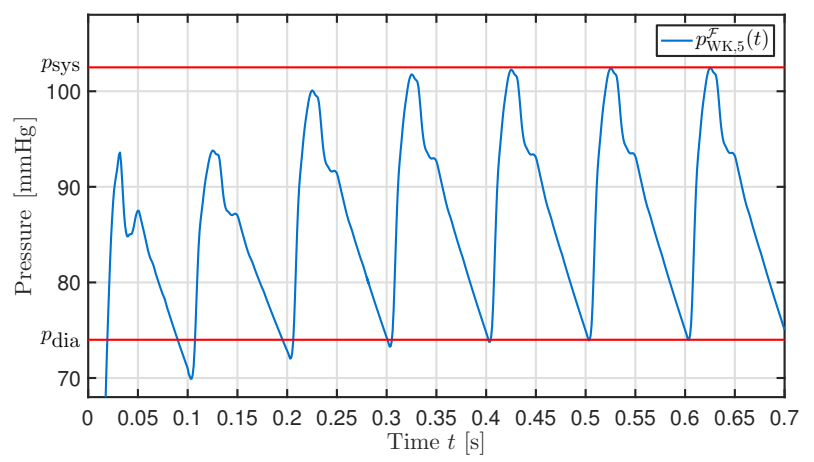

Fig. 9 Pressure from three-element Windkessel on outflow $\Gamma_{\text {Out }, 5}$ over time $t$. After approximately four cardiac cycles the periodic state with the diastolic pressure $p_{\text {dia }}=74.0 \mathrm{mmHg}$ and the systolic pressure $p_{\mathrm{sys}}=102.5 \mathrm{mmHg}$ is reached.

of interest here, namely WSS and PSF have only negligible sensitivity to the absolute hydrostatic pressure level due to the applied prestressing procedure. Therefore, the impact of higher pressure levels to the presented results was computationally investigated (results not shown) showing that they are only minor affected. The qualitative shape of the pressure over time is in good agreement with the results achieved in [77].

As a result of the fluid pressure and the prestressing of the structure, the structure undergoes a maximal radial enlargement of the inner artery wall of around $14-17 \%$ in the aortic arch and around $8-12 \%$ in its branches, which is in good agreement to the measurements in [67]. Thus, the distensibilities (i.e. the radial enlargements per pressure increase) of the aortic arch and its branches are around $5-6 \cdot 10^{-3} \frac{1}{\mathrm{mmHg}}$ and $3-4 \cdot 10^{-3} \frac{1}{\mathrm{mmHg}}$, respectively, lying in the same order of magnitude as reported in [4].

Due to the pulsatile fluid flow the instantaneous WSS $\tau^{\mathcal{F}}$ changes rapidly over time. But since the WSS dependent migration of LDL into the artery wall is on a much larger time scale than the cardiac cycle, it is convenient to look at the time-averaged $\operatorname{WSS}<\boldsymbol{\tau}>^{\mathcal{F}}$, where we have dropped the time index to ease notation. If not explicitly stated otherwise the time-average is over the seventh simulated cardiac cycle. The estimated reference WSS $\left\|\overline{\boldsymbol{\tau}}^{\mathcal{F}}\right\|=6.404 \mathrm{~Pa}$ (see equation (65)) lies in perfect agreement with measurements from literature [15]. The norm of the peak of the computed time-averaged WSS $\left\|\left\langle\boldsymbol{\tau}^{\mathcal{F}}\right\rangle\right\|$ is $49.28 \mathrm{~Pa}$ which corresponds to the 7.7 times of the norm of the reference WSS $\left\|\overline{\boldsymbol{\tau}}^{\mathcal{F}}\right\|$, both lying in good agreement with computational results from literature [29,94]. When the instantaneous WSS $\boldsymbol{\tau}^{\mathcal{F}}$ is used in equation (67) the instantaneous PSF $s\left(\left\|\boldsymbol{\tau}^{\mathcal{F}}\right\|\right)$ is computed. However, in experiments the long time behavior is measured, which is determined by the mean of the PSF . Therefore, one can computationally inves- 


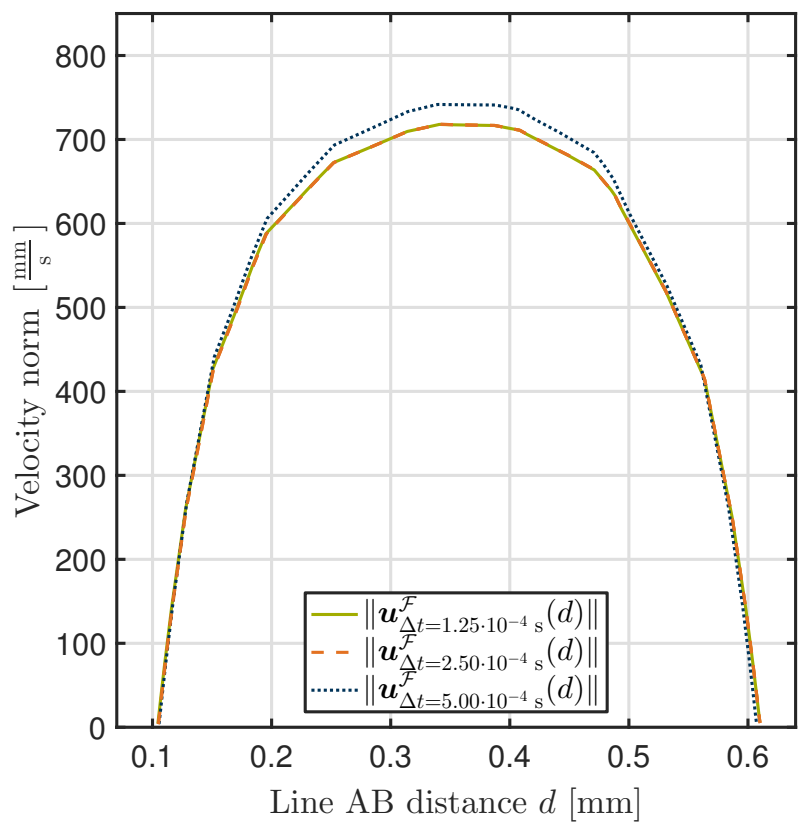

(a) Comparison of the velocitiy profiles over the line $\mathrm{AB}$ (see Figure 4) for different time step sizes $\Delta t$ of $1.25 \cdot 10^{-4} \mathrm{~s}$, $2.5 \cdot 10^{-4} \mathrm{~s}, 5.0 \cdot 10^{-4} \mathrm{~s}$ and a mesh with characteristic element length $h=0.06 \mathrm{~mm}$ at time $t=0.412 \mathrm{~s}$.

Fig. 8 Temporal convergence study.

tigate three different scenarios: the time-average of the instantaneous PSF $<s\left(\left\|\boldsymbol{\tau}^{\mathcal{F}}\right\|\right)>$, the PSF of the timeaveraged norm of the WSS $s\left(<\left\|\boldsymbol{\tau}^{\mathcal{F}}\right\|>\right)$ and the PSF of the norm of the time-averaged WSS $s\left(\left\|<\boldsymbol{\tau}^{\mathcal{F}}>\right\|\right)$. The computational study of these three cases showed that only the latter case is able to match observations from literature $[69,29]$. The second case did produce a qualitatively but not quantitatively correct PSF pattern and the first did result in a more or less homogeneous PSF. In the following we call $\left.s\left(\|<\boldsymbol{\tau}^{\mathcal{F}}\right\rangle \|\right)$ the time-averaged PSF to ease the language. It is visualized from the anterior and posterior view in Figures 10(a) and 10(b), respectively. The time-averaged PSF varies in the range between 0.18 and 4.74 . Hence, the computed scalings differ by a factor of 26.3 showing very good agreement to the measurements in [42].

As visible in Figure 10(a) the simulated regions with high endothelial permeability and hence high risk for atherosclerotic driven plaque development are located at the ascending aorta, near bifurcations and at the bottom and side of the aortic arch. This qualitative phenomena was also observed in experiments $[69,3]$ and computational results by others [19,29,8,94]. Further, is the location of atherosclerotic plaques at theses sites qualitatively supported by our own experimental observations with atherosclerotic mice models, see Figure 11.

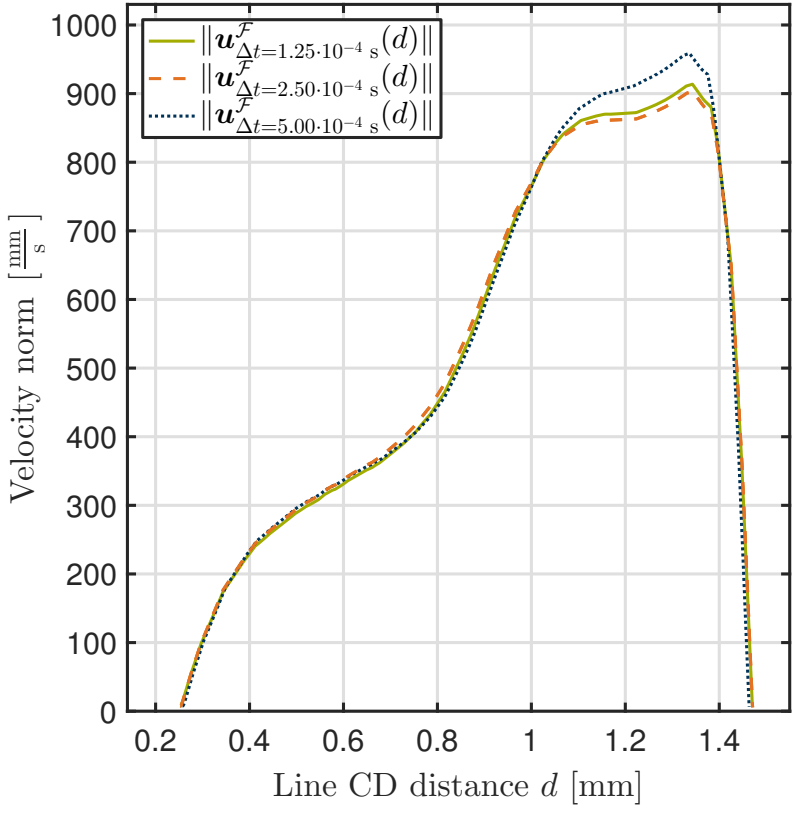

(b) Comparison of the velocitiy profiles over the line CD (see Figure 4) for different time step sizes $\Delta t$ of $1.25 \cdot 10^{-4} \mathrm{~s}$, $2.5 \cdot 10^{-4} \mathrm{~s}, 5.0 \cdot 10^{-4} \mathrm{~s}$ and a mesh with characteristic element length $h=0.06 \mathrm{~mm}$ at time $t=0.412 \mathrm{~s}$.

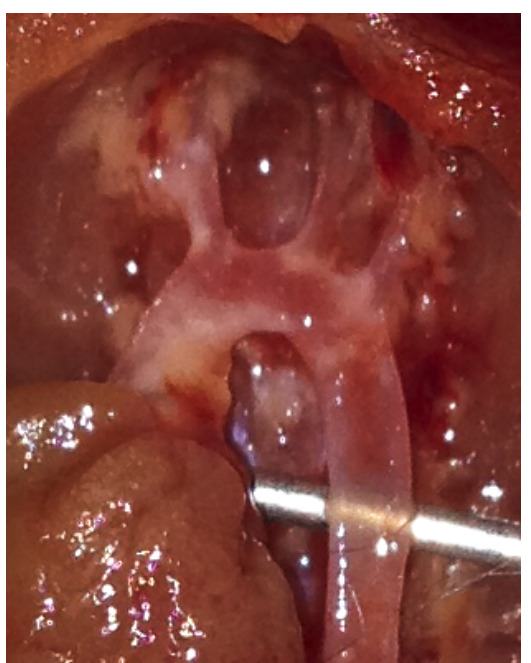

Fig. 11 Dissection of an aortic arch of a LDL receptor deficient mouse (type B6.129S7-Ldlrtm1Her/J) with atherosclerotic plaques (white).

Remark. Even if not explicitly considered in the computation of the PSF the spatial distribution of the OSI $o\left(\boldsymbol{\tau}^{\mathcal{F}}\right)=\frac{1}{2}\left(1-\frac{\left\|<\boldsymbol{\tau}^{\mathcal{F}}>\right\|}{<\left\|\boldsymbol{\tau}^{\mathcal{F}}\right\|>}\right)[43,80,89]$ is plotted from two perspectives in Figures 10(c) and 10(d). An OSI of zero corresponds to regions of non-oscillatory and an OSI of 0.5 to regions of highly oscillatory flow. As visible the pattern is comparable to the computed PSF visualized in Figures 10(a) and 10(b). This supports 


$$
\begin{aligned}
& \text { time-averaged PSF } s\left(\left\|<\boldsymbol{\tau}^{\mathcal{F}}>\right\|\right)[1] \\
& 0.18 \\
& 4.74
\end{aligned}
$$
0.18 4.74

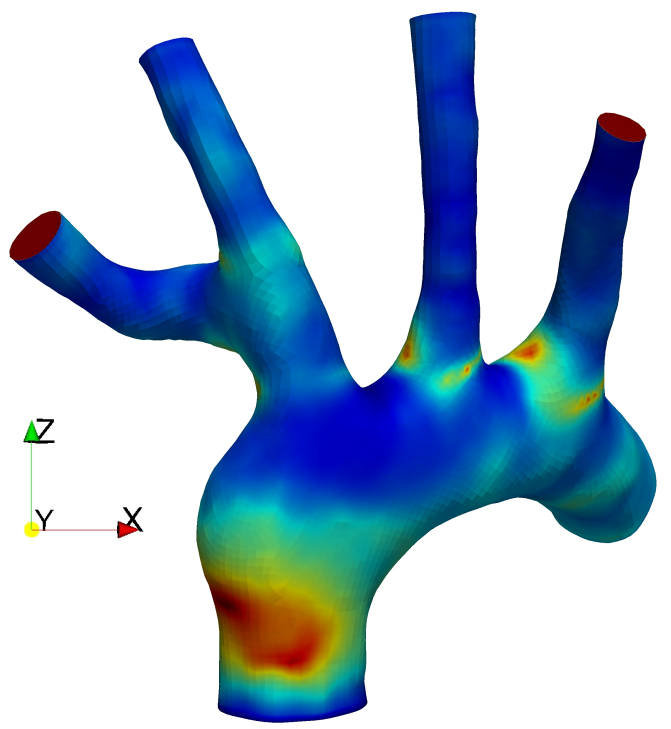

(a) Anterior view of the spatial distribution of time-averaged permeability scaling factor $s\left(\left\|<\tau^{\mathcal{F}}>\right\|\right)$.

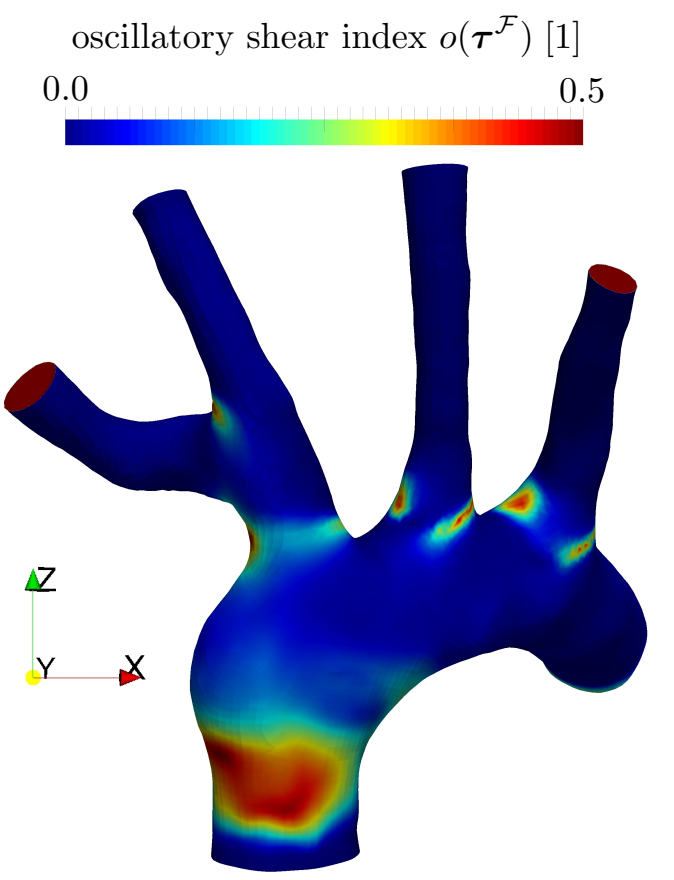

(c) Anterior view of the spatial distribution of oscillatory shear index $o\left(\boldsymbol{\tau}^{\mathcal{F}}\right)$.

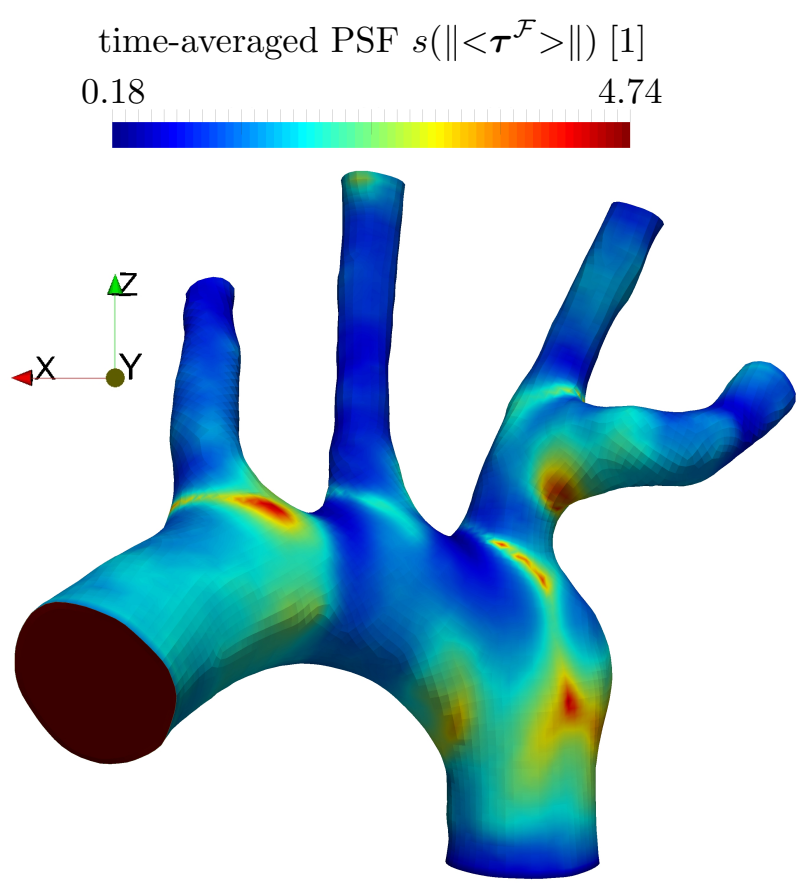

(b) Posterior view of the spatial distribution of timeaveraged permeability scaling factor $s\left(\left\|<\boldsymbol{\tau}^{\mathcal{F}}>\right\|\right)$.
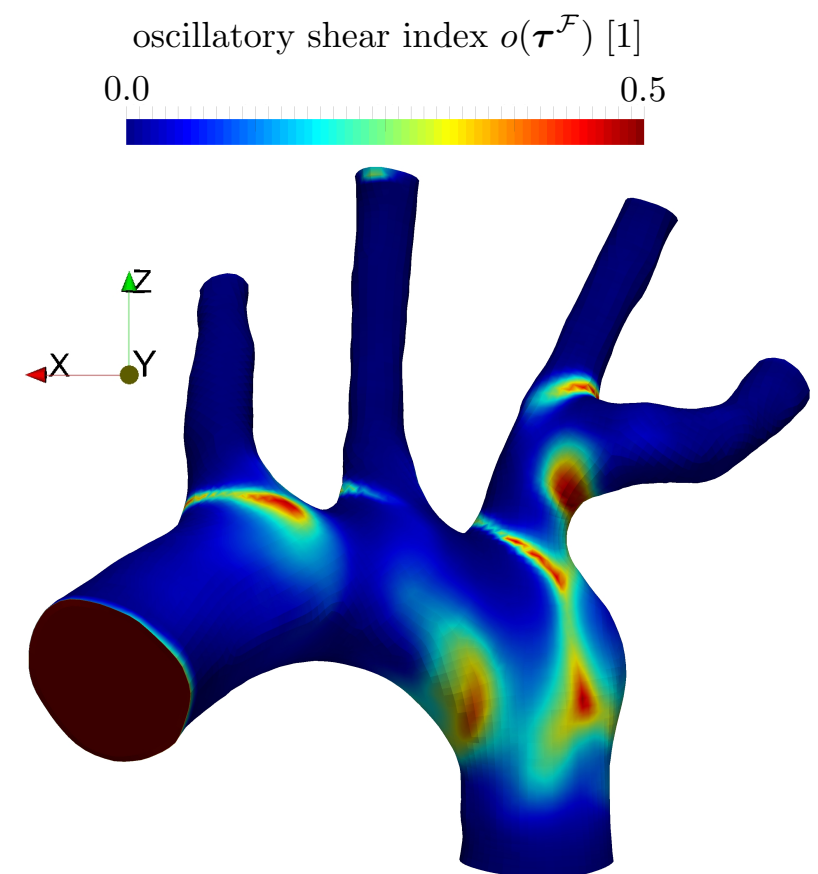

(d) Posterior view of the spatial distribution of oscillatory shear index $o\left(\boldsymbol{\tau}^{\mathcal{F}}\right)$.

Fig. 10 Spatial distribution of the time-averaged permeability scaling factor and oscillatory shear index. 
the theory that the OSI may also be a valid indicator for atherosclerosis plaque localization as is critically discussed in literature, see [80] and therein. But since the actual influence of the OSI to the endothelial permeability is unknown it is not explicitly considered in the presented model and is subject to future research.

4.5 Influence of the compliance of the structure and the pulsatile flow

We study the influence of model reductions frequently found in literature. Therefore, the PSF derived from the present model with the PSF of reduced models is compared. As model reductions we assume two simplified versions of the presented model.

The first reduced model is the case of a non-compliant artery wall, resulting in a rigid wall model. This simplification is enforced by the addition of the condition $\mathbf{d}^{\mathcal{S}}=\mathbf{0}$ compared to the full model. Such types of models are frequently proposed, especially in the context of porous media models of the artery wall, see e.g. [81,106,11,66,93]. The computed time-averaged PSF $\left.s\left(\|<\boldsymbol{\tau}^{\mathcal{F}}\right\rangle \|\right)$ of the rigid wall model is visualized in Figure 12(a). The computed OSI $o\left(\boldsymbol{\tau}^{\mathcal{F}}\right)$ for the rigid wall model is visualized in Figure 12(c).

The second reduced model scenario frequently found in literature is the case of time-averaged, stationary flows $[99,51,96,11,71]$. This simplification is achieved by assuming the influx to be constant in time, i.e. by $Q_{\text {In }}^{\mathcal{F}}(t)=\bar{Q}_{\text {In }}^{\mathcal{F}}$. As consequence, the pressure of the fluid determined by the Windkessel subproblems is constant and no displacements can be expected from the loaded in vivo state. Hence, the time-averaging of flows implies $\mathbf{d}^{\mathcal{S}}=0$. The computed PSF $s\left(\left\|<\boldsymbol{\tau}^{\mathcal{F}}>\right\|\right)$ of the timeaveraged flow model is visualized in Figure 12(b). The computed OSI $o\left(\boldsymbol{\tau}^{\mathcal{F}}\right)$ for the time-averaged flow model is visualized in Figure 12(d).

\subsection{Growth and remodeling}

To show the capability of the cardiovascular model to adapt to the narrowing of the lumen due to the foam cell accumulation we compare the spatial distribution of the PSF at different times. Since bio-chemical reactions and growth and remodeling processes take place on a much larger time scale as the hemodynamics (compare e.g. the orders of the length of the cardiac cycle $T_{\mathrm{Cycl}}$ and the diffuse permeability $P_{D}$ ) and as the model is not embedded into a multiscale strategy, some model parameters are increased to accelerate atherosclerosis progression to the duration of a few cardiac cycles. Hence, after the cardiovascular model obtained its periodic state the following adapted model parameters were used: $D_{\mathrm{LDL}}^{\mathcal{S S}}=6.0 \cdot 10^{-2} \frac{\mathrm{mm}^{2}}{\mathrm{~s}}, d_{\mathrm{LDL}}^{\mathcal{S S}}=1.0 \frac{1}{\mathrm{~s}}, \gamma_{\mathrm{LDL}}^{\mathcal{S S}}=0.4 \frac{1}{\mathrm{~s}}$, $c_{\mathrm{LDL}, \text { Thres }}^{\mathcal{S S}}=2.0 \cdot 10^{-3} \frac{\mathrm{s}}{\mathrm{LDL}, \mathrm{In}}, P_{D}=5.0 \cdot 10^{-4} \frac{\mathrm{mm}}{\mathrm{s}}$, $k_{\text {Wall }}^{\mathcal{S}}=1.0 \cdot 10^{6} \frac{\mathrm{Pa}}{\mathrm{mm}}$ and $\alpha=4.1 \cdot 10^{10} \frac{\mathrm{mm}^{3}}{\mathrm{~g}}$. In Figures $13(\mathrm{a})$ and $13(\mathrm{~b})$ the time-averaged PSF of the grown artery wall at different times are visualized. Figures 13(c), 14(b) and 14(c) show the growth and remodeling factors of the grown artery wall at time $t=1.2 \mathrm{~s}$.

\section{Discussion}

We have presented a methodology to calibrate our model to a specific geometry and a given set of key physiological data. The validation of the model in section 4.4 showed that computed key physiological quantities such as blood pressure, artery wall displacements and WSS derived from the considered exemplary set of murine physiological data are qualitatively in good agreement with measurements and simulations performed by others. However, there are large inter- and intramouse variations of these quantities depending on the condition, type, age or size of the specific mouse and its geometry, see e.g. [15,103,63] and therein. Still, a quantitative validation of the developed model remains to be done, once a complete in vivo data-set is available to us.

The newly developed calibration of the time-averaged WSS dependent law for the scaling of the endothelial permeability $\left.s\left(\|<\boldsymbol{\tau}^{\mathcal{F}}\right\rangle \|\right)$ to the specific geometry can be used to estimate potential plaque locations. The predicted plaque locations are geometry-driven and thus only a qualitative agreement with other studies could be found. A quantitative validation of the developed PSF for the specific geometry remains to be done. We plan to image the in vivo plaque locations and compare them to the predicted locations by the PSF in terms of a suitable metric, see e.g. the work in [21]. A picture of a dissection of a murine aortic arch with atherosclerotic plaques is given in Figure 11. The alternative formulations for the time-averaged endothelial PSF $<s\left(\left\|\boldsymbol{\tau}^{\mathcal{F}}\right\|\right)>$ and $s\left(\left\langle\left\|\boldsymbol{\tau}^{\mathcal{F}}\right\|>\right)\right.$ did not result in physiologically meaningful results.

In literature, models of atherosclerosis with timeaveraged flows are commonly utilized. This is often argued by the idea that the growth and remodeling process in atherosclerosis is on a much larger time scale than the hemodynamics and hence also the mean blood flow is a right indicator for the mechanobiology behind. In contrast to this argument, the comparison of our proposed model with the reduced time-averaged flow model introduced in section 4.5, shows a significant difference in the WSS pattern and hence their time-averaged PSF pattern, see Figures 10(a) and 12(b). This observation is 


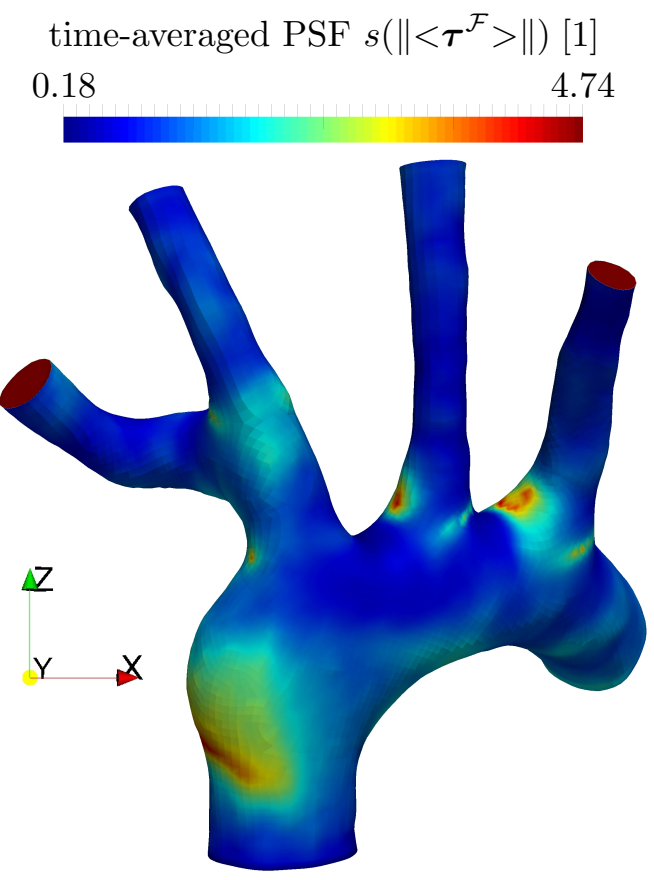

(a) Anterior view of the spatial distribution of time-averaged permeability scaling factor $s\left(\left\|<\tau^{\mathcal{F}}>\right\|\right)$ of the rigid wall model.

oscillatory shear index $o\left(\boldsymbol{\tau}^{\mathcal{F}}\right)[1]$

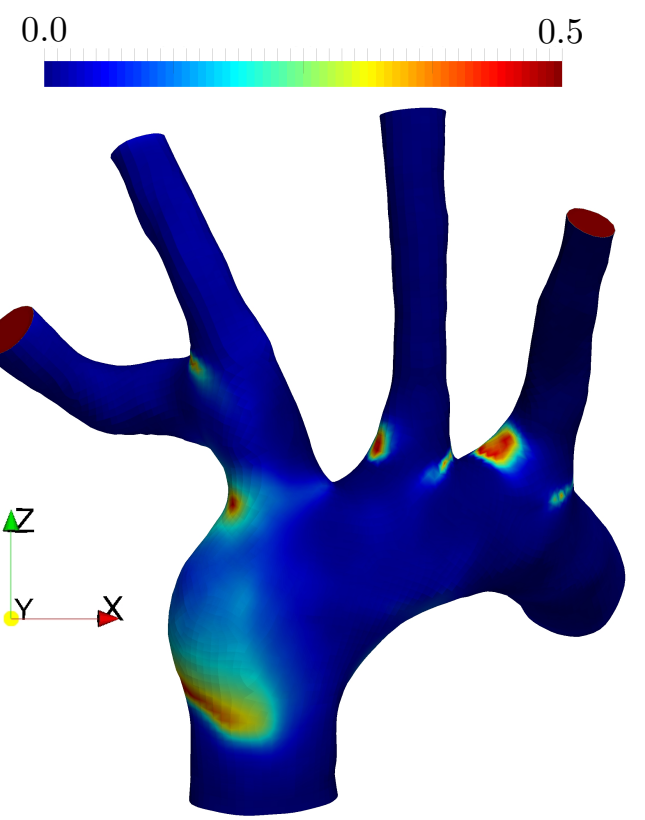

(c) Anterior view of the spatial distribution of the oscillatory shear index $o\left(\tau^{\mathcal{F}}\right)$ of the rigid wall model.

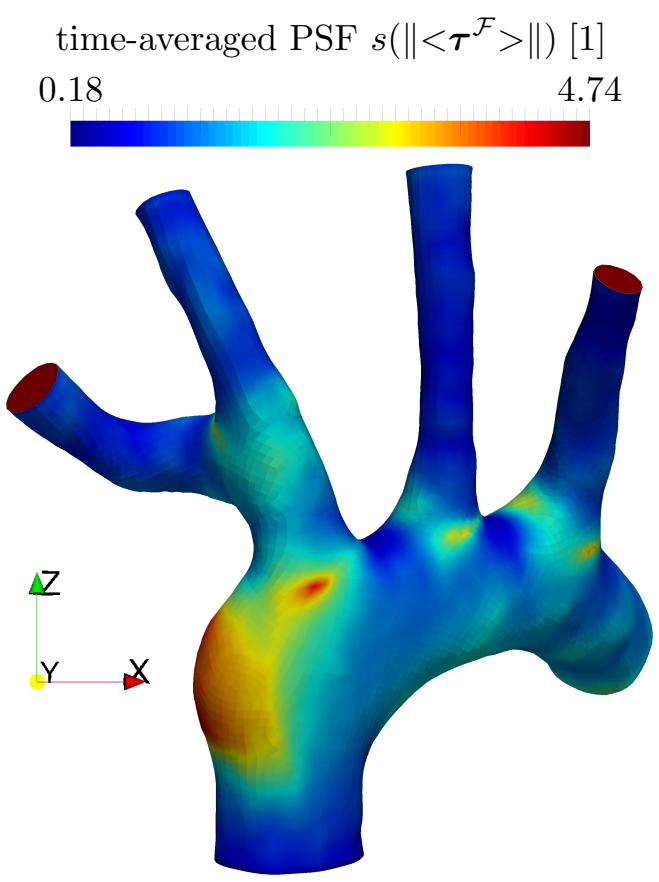

(b) Anterior view of the spatial distribution of time-averaged permeability scaling factor $s\left(\left\|<\boldsymbol{\tau}^{\mathcal{F}}>\right\|\right)$ of the time-averaged flow model.

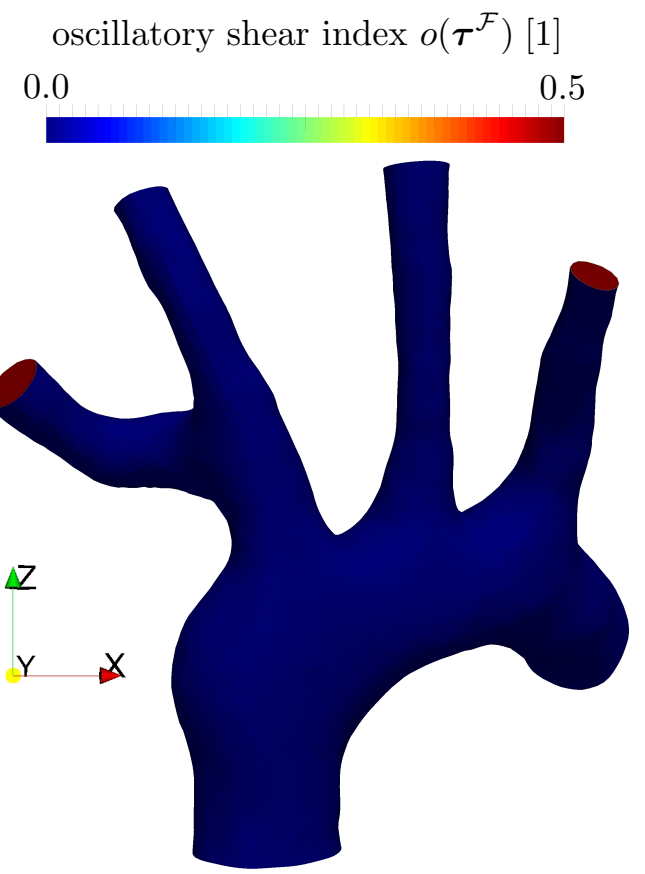

(d) Anterior view of the spatial distribution of the oscillatory shear index $o\left(\boldsymbol{\tau}^{\mathcal{F}}\right)$ of the time-averaged flow model.

Fig. 12 Spatial distribution of time-averaged permeability scaling factor and oscillatory shear index for the rigid and mean-flow model. 
time-averaged PSF $s\left(\left\|<\boldsymbol{\tau}^{\mathcal{F}}>_{0.5} \mathrm{~s}\right\|\right)[1]$

0.18

4.74

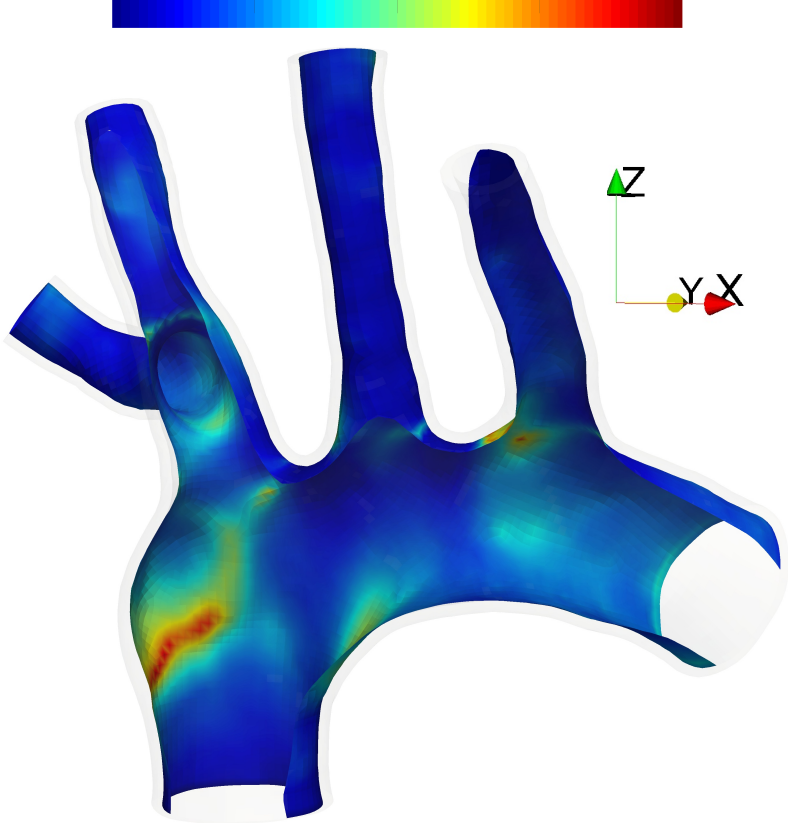

(a) Spatial distribution of time-averaged permeability scaling factor $s\left(\left\|<\tau^{\mathcal{F}}>_{0.5} \mathrm{~s}\right\|\right)$ at time $t=0.5 \mathrm{~s}$. time-averaged PSF $s\left(\left\|<\boldsymbol{\tau}^{\mathcal{F}}>_{1.2 \mathrm{~s}}\right\|\right)[1]$

0.18

4.74

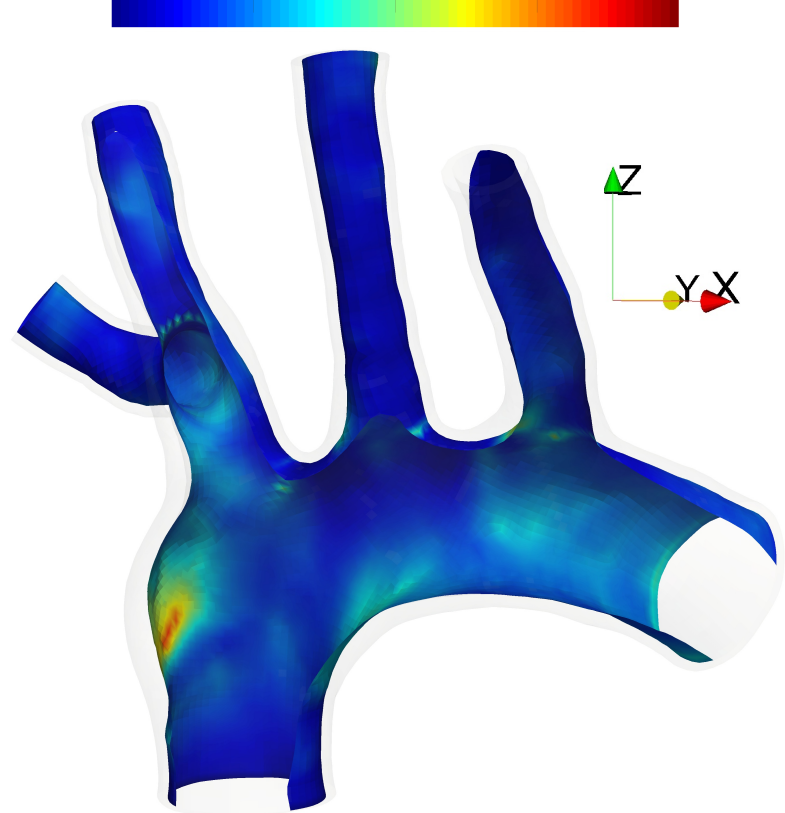

(b) Spatial distribution of time-averaged permeability scaling factor $s\left(\left\|<\tau^{\mathcal{F}}>_{1.2} \mathrm{~s}\right\|\right)$ at time $t=1.2 \mathrm{~s}$.

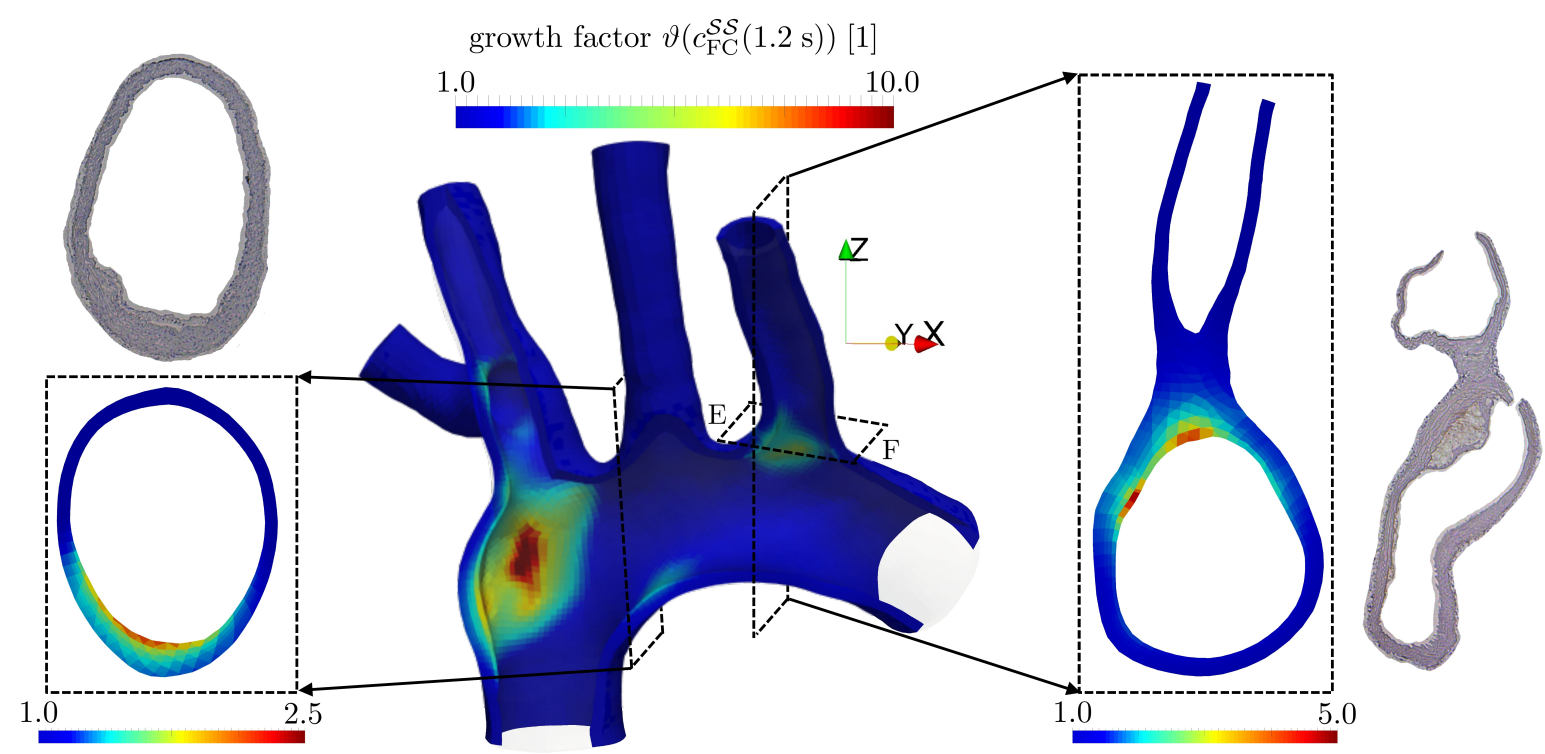

(c) Grown artery wall, spatial distribution of growth factor $\vartheta\left(c_{\mathrm{F}}^{\mathcal{S}} \mathcal{S}(1.2 \mathrm{~s})\right)$ at time $t=1.2 \mathrm{~s}$ and comparison of grown cross sections with aortic cross sections from LDL receptor deficient mice (type B6.129S7-Ldlrtm1Her/J) with early (left top) and advanced (right) atherosclerotic plaques. The murine cross sections were stained with haematoxylin.

Fig. 13 Cross sections of spatial distribution of time-averaged permeability scaling factor and growth factor at different times and comparison of grown cross sections with mouse experiments. 
even more valid when comparing the OSI computed by the two models, see Figures 10(c) and 12(d). This effect is due to the unphysiological averaging of the pulsatile nature of blood flow preventing flow recirculations and oscillatory flows frequently occurring in the diastolic phase of the cardiac cycle, where the cardiac output is close to zero. In the case of time-averaged flows we do not observe such oscillatory flows at all, see Figure $12(\mathrm{~d})$. This observation strongly supports the theory that the above described model reduction of neglecting the pulsatile nature of blood flow and hence using a time-averaged flow is misleading, as is stated by others too $[66,93,29,58]$.

In contrast, the comparison of results of the full model with its rigid wall simplification shows that the displacements of the artery wall only have a minor influence on the WSS and PSF patterns, see Figures 10(a) and 12(a). Similar observations are also made for the computed OSI of the two models, see Figures 10(c) and $12(\mathrm{c})$. In our case study we find that the timeaveraged WSS and OSI and hence the endothelial permeability is only slightly affected by radial enlargements. Hence, the rigid wall model is also capable to properly compute the spatial distribution of the time-averaged PSF and the OSI patterns and hence is also suited to predict the potential plaque development locations. The difference in the PSF mainly is that the non-compliant artery wall model yields sharper transitions between low and high permeability regions, where in contrast the compliant artery wall does produce broader and more blurred high permeability regions. Similar observations for the WSS patterns in carotid arteries were observed in [20]. However, in [20] a significant influence of the compliant artery wall to the OSI was observed which lies in contrast to our observations.

Still we also support the idea that a FSI-like approach to model atherosclerosis is indispensable. This is also stated by others $[58,20,21]$, but the reason for our conclusion is different. Our observations indicate that not the radial enlargement of the lumen arises the need for a FSI approach, but much more the ability of the model to capture the permanent displacements of the endothelium due to the artery wall thickening. Consequently, a classical FSI approach is not mandatory, but mainly a rigid wall and pulsatile flow model where the vessel lumen adapts to growth and remodeling. Still, a pure CFD simulation is not sufficient since growth and remodeling processes can only be captured in a physiological manner when the specific layout of the artery wall, i.e. a suitable constitutive law is considered. Hence, a FSI-like approach is indispensable to physiologically capture the influences of the large time scale phenomena in atherosclerosis to the cardiovascular mechanics.
To accelerate the development of atherosclerotic plaques to a few cardiac cycles some parameters of the model had to be adapted. This was done in a way such that the developed plaques qualitatively match plaques found in our experiments with atherosclerotic mice models, see Figure 13(c).

As indicated in Figures 13(a) and 13(b), growth successively narrows the lumen and induces a drastic change of the PSF pattern representing the endothelial permeability. To give a quantitative example, the luminal area of cross section EF (see Figure 13(c)) is reduced by $36,8 \%$ compared to the initial state, see Figure 14 . Even if the results were achieved with our simplistic inflammatory model, this illustrates the presented model of the cardiovascular mechanics is capable to adjust dynamically to the large time scale atherosclerotic process of growth and remodeling. For the primary high permeability regions with already developed plaques there are two main trends. On the one hand can the endothelial permeability could decrease such that the healing processes outweigh the continuing LDL penetration and hence the atherosclerotic process locally stagnates resulting in a stable plaque. In the contrary case, the endothelial permeability would be too high for the plaque to become stable and the plaque continues to grow. To be able to predict the stability outcome of a plaque, a predictive reaction model for the species must be established to replace our simplified model. However, such a mathematical model is yet not developed. Further, it is inevitable to embed the presented model into a suitable multiscale strategy as e.g. presented in [31].

\section{Limitations}

The presented model is affected by five main limitations. First, the conclusions drawn from the developed mathematical and computational model are based only on a single murine-specific computational case study which is based on a single set of physiological data from literature. Hence, the murine-specific setup in our model only differs in the geometry and geometry-derived model parameters. Still, the computational case study of our model showed that our proposed model is well applicable in the context of atherosclerosis. We expect that the qualitatively same results are achieved also for other murine-specific geometries and derived parameter sets. In further work more murine-specific cases will be solved to further confirm the presented conclusions.

Second, the reaction model is a drastic simplification of the complex bio-chemical processes in atherosclerosis. Many important processes and key species involved in the development of atherosclerotic plaques were neglected and a simple heuristic reaction model with solely 

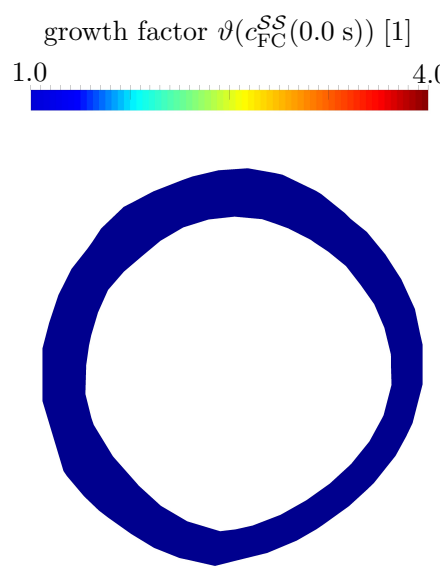

(a) Initial cross section $\mathrm{EF}$ and spatial distribution of growth factor $\vartheta\left(c_{\mathrm{FC}}^{\mathcal{S}}(0.0 \mathrm{~s})\right)$ at time $t=0.0 \mathrm{~s}$.
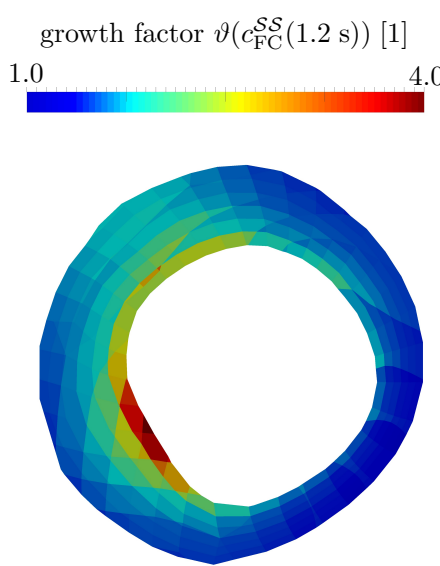

(b) Grown cross section EF and spatial distribution of growth factor $\vartheta\left(c_{\mathrm{FC}}^{\mathcal{S}}(1.2 \mathrm{~s})\right)$ at time $t=1.2 \mathrm{~s}$.

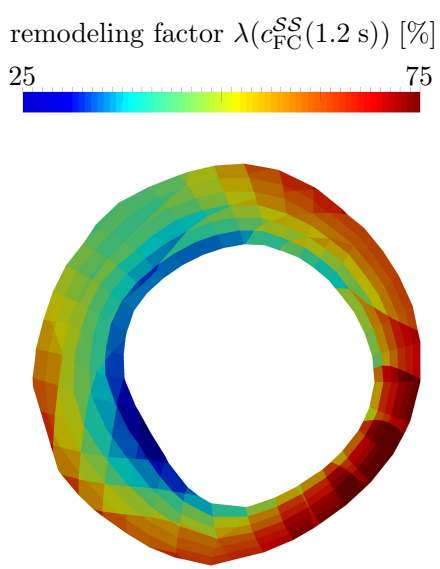

(c) Grown cross section EF and spatial distribution of remodeling factor $\lambda\left(c_{\mathrm{FC}}^{\mathcal{S}}(1.2 \mathrm{~s})\right)$ at time $t=1.2 \mathrm{~s}$.

Fig. 14 Initial and grown cross section EF (compare Figure 13(c)) and spatial distribution of growth factor and remodelling factor at different times.

two species (LDL and foam cells) was utilized. However, a quantitatively validated and predictive model for the development of early atherosclerotic plaques is yet not available and hence more sophisticated reaction models from literature would not increase the validity of our computational results. The development of such a predictive model and its implementation into the present model is ongoing work.

The third limitation of the proposed model is its computational cost preventing it from a straightforward application to predict the long time process of atherosclerosis. In future work the model has to be embedded into a suitable multiscale in time strategy, e.g. similar to [31]. Thereby, the presented models of cardiovascular mechanics and concentrations in the blood can be utilized on the small time scale. In contrast, the model of concentrations in the artery wall (with a more sophisticated reaction model) can be used on the large time scale. As has been shown here, the modeling of the small time scale is indispensable and hence the usage of a multiscale in time strategy is unavoidable.

Another limitation of the model is that it suffers from a variety of uncertainties which were yet not assessed. The utilized magnetic resonance angiography has a rather coarse spatial resolution (especially throughplane) compared to other imaging techniques like micro computer tomography. Thus, due to the common sequence of imaging, segmentation and simulation, immanent inaccuracies in the segmented geometry of the lumen may result in geometry-driven alterations of computed results, especially of the WSS and PSF patterns [63]. Additionally, the coarse resolution did not allow for a segmentation of the artery wall thickness and thus the simplification of a constant wall thickness was employed. Further, some model parameters are only roughly known as they are very difficult to measure (such as the spatial variation of the diffusive permeability) or were not yet measured based on murine experiments (such as the parameters for the surrounding tissue). A detailed quantification of theses uncertainties in our model is very challenging and must be individually addressed in future work.

Finally, the neglection of the transmural pressure gradient driven porous media flow inside the artery wall and the subsequent neglection of the convective solute flux through the endothelium is another limitation. But since the mechanical properties are dominated by the adventitia and media layers of the artery wall and these layers mainly consist of a solid phase [106] the influence of the fluid phase to the cardiovascular mechanics here is assumed to be minor. Additionally, the importance of the porous media flow on the transportation of LDL through the endothelium is controversial, see e.g. [97] and [75], and requires further investigations.

\section{Conclusion}

A novel mathematical multiphysics model of early atherosclerosis has been proposed. It consists of a fluidstructure interaction model of cardiovascular mechanics including a novel scalar dependent anisotropic growth and remodeling formulation. Further, an advection-diffusion-reaction model including a law for the endothelial permeability was introduced to account for the species transport in the blood and their migration into and reaction in the artery wall. 
It was shown how the model can be calibrated to given physiological data sets and murine-specific geometries such that it reproduces important cardiovascular quantities such as the blood pressure, radial displacements, wall shear stresses and others. The novel law for the up- and downscaling of the endothelial permeability with respect to LDL proved to be a good indicator for potential atherosclerotic plaque development. The analysis of our computational case study further supports the theory that neglecting the small time scale of cardiovascular mechanics in terms of averaging flows and neglecting the deformation of the artery wall is misleading in the context of atherosclerosis. It was shown that successive growth influences the wall-shear stress pattern and hence a dynamical adjustment of the LDL penetration is induced. In future work, the present multiphysics model must be enriched by a more sophisticated model of the inflammatory processes. Additionally, it must be embedded into a suitable multi-scale in time strategy.

Acknowledgements The authors gratefully acknowledge the financial support given by the International Graduate School of Science and Engineering of the Technical University of Munich under the project BioMat01, A Multiscale Model of Atherosclerosis. This work was supported by the German Research Foundation (DFG) and the Technical University of Munich (TUM) in the framework of the Open Access Publishing Program.

Conflict of Interest All authors declare that no conflicts of interest exist.

\section{References}

1. Ambrosi, D., Mollica, F.: On the mechanics of a growing tumor. International Journal of Engineering Science 40(12), 1297-1316 (2002)

2. Arora, D.: Computational hemodynamics: Hemolysis and viscoelasticity. Ph.D. thesis, Citeseer (2005)

3. Asakura, T., Karino, T.: Flow patterns and spatial distribution of atherosclerotic lesions in human coronary arteries. Circulation Research 66(4), 1045-1066 (1990)

4. Aslanidou, L., Trachet, B., Reymond, P., Fraga-Silva, R.A., Segers, P., Stergiopulos, N.: A 1d model of the arterial circulation in mice. ALTEX 33(1), 13 (2016)

5. Balzani, D., Schmidt, T.: Comparative analysis of damage functions for soft tissues: Properties at damage initialization. Mathematics and Mechanics of Solids 20(4), 480-492 (2015)

6. Barrenechea, G.R., Valentin, F.: An unusual stabilized finite element method for a generalized stokes problem. Numerische Mathematik 92(4), 653-677 (2002)

7. Bazilevs, Y., Calo, V.M., Tezduyar, T.E., Hughes, T.J.: $\mathrm{YZ} \beta$ discontinuity capturing for advection-dominated processes with application to arterial drug delivery. International Journal for Numerical Methods in Fluids 54, 593-608 (2007)
8. Bazilevs, Y., Gohean, J., Hughes, T., Moser, R., Zhang, Y.: Patient-specific isogeometric fluid-structure interaction analysis of thoracic aortic blood flow due to implantation of the jarvik 2000 left ventricular assist device. Computer Methods in Applied Mechanics and Engineering 198(45), 3534-3550 (2009)

9. Bird, R.B., Armstrong, R., Hassager, O.: Dynamics of polymeric liquids. vol. 1: Fluid mechanics (1987)

10. Brown, A.J., Teng, Z., Evans, P.C., Gillard, J.H., Samady, H., Bennett, M.R.: Role of biomechanical forces in the natural history of coronary atherosclerosis. Nature Reviews Cardiology (2016)

11. Calvez, V., Houot, J.G., Meunier, N., Raoult, A., Rusnakova, G.: Mathematical and numerical modeling of early atherosclerotic lesions. ESAIM: Proceedings and Surveys 30, 14 (2010). DOI 10.1051/proc/2010002

12. Carew, T.E., VAISHNAV, R.N., PATEL, D.J.: Compressibility of the arterial wall. Circulation Research 23(1), 61-68 (1968)

13. Chalmers, A.D., Cohen, A., Bursill, C.A., Myerscough, M.R.: Bifurcation and dynamics in a mathematical model of early atherosclerosis. Journal of Mathematical Biology 71(6-7), 1451-1480 (2015)

14. Chen, J., Lu, X.Y.: Numerical investigation of the nonnewtonian pulsatile blood flow in a bifurcation model with a non-planar branch. Journal of Biomechanics 39(5), 818-832 (2006)

15. Cheng, C., Helderman, F., Tempel, D., Segers, D., Hierck, B., Poelmann, R., van Tol, A., Duncker, D.J., RobbersVisser, D., Ursem, N.T., et al.: Large variations in absolute wall shear stress levels within one species and between species. Atherosclerosis 195(2), 225-235 (2007)

16. Cho, Y.I., Kensey, K.R.: Effects of the non-newtonian viscosity of blood on flows in a diseased arterial vessel. part 1: Steady flows. Biorheology (28), 241-62 (1991)

17. Cilla, M., Peña, E., Martínez, M.A.: Mathematical modelling of atheroma plaque formation and development in coronary arteries. Journal of The Royal Society Interface 11(90), 20130,866 (2014)

18. Codina, R.: Stabilized finite element approximation of transient incompressible flows using orthogonal subscales. Computer Methods in Applied Mechanics and Engineering 191(39), 4295-4321 (2002)

19. Crosetto, P., Reymond, P., Deparis, S., Kontaxakis, D., Stergiopulos, N., Quarteroni, A.: Fluid-structure interaction simulation of aortic blood flow. Computers \& Fluids 43(1), 46-57 (2011)

20. De Wilde, D., Trachet, B., De Meyer, G., Segers, P.: The influence of anesthesia and fluid-structure interaction on simulated shear stress patterns in the carotid bifurcation of mice. Journal of Biomechanics 49(13), 2741-2747 (2016)

21. De Wilde, D., Trachet, B., De Meyer, G.R., Segers, P.: Shear stress metrics and their relation to atherosclerosis: An in vivo follow-up study in atherosclerotic mice. Annals of Biomedical Engineering pp. 1-12 (2015)

22. De Wilde, D., Trachet, B., Debusschere, N., Iannaccone, F., Swillens, A., Degroote, J., Vierendeels, J., De Meyer, G.R., Segers, P.: Assessment of shear stress related parameters in the carotid bifurcation using mouse-specific fsi simulations. Journal of Biomechanics (2015)

23. Dobrin, P.B., Rovick, A.A.: Influence of vascular smooth muscle on contractile mechanics and elasticity of arteries. American Journal of Physiology-Legacy Content 217(6), 1644-1651 (1969)

24. Doll, S., Schweizerhof, K.: On the development of volumetric strain energy functions. Journal of Applied Mechanics 67(1), 17-21 (2000) 
25. Doll, S., Schweizerhof, K., Hauptmann, R., Freischläger, C.: On volumetric locking of low-order solid and solidshell elements for finite elastoviscoplastic deformations and selective reduced integration. Engineering Computations 17(7), 874-902 (2000)

26. Donea, J., Giuliani, S., Halleux, J.: An arbitrary lagrangian-eulerian finite element method for transient dynamic fluid-structure interactions. Computer Methods in Applied Mechanics and Engineering 33(1), 689-723 (1982)

27. Donea, J., Huerta, A.: Finite element methods for flow problems. John Wiley and Sons (2003)

28. Faxon, D.P., Fuster, V., Libby, P., Beckman, J.A., Hiatt, W.R., Thompson, R.W., Topper, J.N., Annex, B.H., Rundback, J.H., Fabunmi, R.P., et al.: Atherosclerotic vascular disease conference writing group iii: Pathophysiology. Circulation 109(21), 2617-2625 (2004)

29. Feintuch, A., Ruengsakulrach, P., Lin, A., Zhang, J., Zhou, Y.Q., Bishop, J., Davidson, L., Courtman, D., Foster, F.S., Steinman, D.A., et al.: Hemodynamics in the mouse aortic arch as assessed by mri, ultrasound, and numerical modeling. American Journal of PhysiologyHeart and Circulatory Physiology 292(2), H884-H892 (2007)

30. Ferruzzi, J., Bersi, M., Humphrey, J.: Biomechanical phenotyping of central arteries in health and disease: advantages of and methods for murine models. Annals of Biomedical Engineering 41(7), 1311-1330 (2013)

31. Figueroa, C.A., Baek, S., Taylor, C.A., Humphrey, J.D.: A computational framework for fluid-solid-growth modeling in cardiovascular simulations. Computer Methods in Applied Mechanics and Engineering 198(45-46), 35833602 (2009). DOI 10.1016/j.cma.2008.09.013

32. Filipovic, N., Rosic, M., Tanaskovic, I., Parodi, O., Fotiadis, D.: Computer simulation and experimental analysis of ldl transport in the arteries. In: Engineering in Medicine and Biology Society, EMBC, 2011 Annual International Conference of the IEEE, pp. 195-198. IEEE (2011)

33. Friedewald, W.T., Levy, R.I., Fredrickson, D.S.: Estimation of the concentration of low-density lipoprotein cholesterol in plasma, without use of the preparative ultracentrifuge. Clinical Chemistry 18(6), 499-502 (1972)

34. Friedman, A., Hao, W.: A mathematical model of atherosclerosis with reverse cholesterol transport and associated risk factors. Bulletin of Mathematical Biology 77(5), 758-781 (2015)

35. Gee, M., Förster, C., Wall, W.: A computational strategy for prestressing patient-specific biomechanical problems under finite deformation. International Journal for $\mathrm{Nu}-$ merical Methods in Biomedical Engineering 26(1), 52-72 (2010)

36. Gee, M.W., Küttler, U., Wall, W.A.: Truly monolithic algebraic multigrid for fluid-structure interaction. International Journal for Numerical Methods in Engineering 85(8), 987-1016 (2011)

37. Gijsen, F., Allanic, E., Van de Vosse, F., Janssen, J.: The influence of the non-newtonian properties of blood on the flow in large arteries: unsteady flow in a 90 curved tube. Journal of Biomechanics 32(7), 705-713 (1999)

38. Goriely, A., Amar, M.B.: On the definition and modeling of incremental, cumulative, and continuous growth laws in morphoelasticity. Biomechanics and Modeling in Mechanobiology 6(5), 289-296 (2007)

39. Gravemeier, V., Comerford, A., Yoshihara, L., Ismail, M., Wall, W.A.: A novel formulation for neumann inflow boundary conditions in biomechanics. International Journal for Numerical Methods in Biomedical Engineering 28(5), 560-573 (2012)

40. Haskett, D., Johnson, G., Zhou, A., Utzinger, U., Geest, J.V.: Microstructural and biomechanical alterations of the human aorta as a function of age and location. Biomechanics and Modeling in Mechanobiology 9(6), 725-736 (2010)

41. Heiland, V.M., Forsell, C., Roy, J., Hedin, U., Gasser, T.C.: Identification of carotid plaque tissue properties using an experimental-numerical approach. Journal of the Mechanical Behavior of Biomedical Materials 27, 226-238 (2013)

42. Herrmann, R.A., Malinauskas, R.A., Truskey, G.A.: Characterization of sites with elevated ldl permeability at intercostal, celiac, and iliac branches of the normal rabbit aorta. Arteriosclerosis, Thrombosis, and Vascular Biology 14(2), 313-323 (1994)

43. Himburg, H.A., Grzybowski, D.M., Hazel, A.L., LaMack, J.A., Li, X.M., Friedman, M.H.: Spatial comparison between wall shear stress measures and porcine arterial endothelial permeability. American Journal of PhysiologyHeart and Circulatory Physiology 286(5), H1916-H1922 (2004)

44. Holzapfel, G.A., Gasser, T.C., Ogden, R.W.: A new constitutive framework for arterial wall mechanics and a comparative study of material models. Journal of Elasticity and the Physical Science of Solids 61(1-3), $1-48(2000)$

45. Holzapfel, G.A., Mulvihill, J.J., Cunnane, E.M., Walsh, M.T.: Computational approaches for analyzing the mechanics of atherosclerotic plaques: a review. Journal of Biomechanics 47(4), 859-869 (2014)

46. Hossain, S.S., Hossainy, S.F., Bazilevs, Y., Calo, V.M., Hughes, T.J.: Mathematical modeling of coupled drug and drug-encapsulated nanoparticle transport in patientspecific coronary artery walls. Computational Mechanics 49(2), 213-242 (2012)

47. Humphrey, J.: Cardiovascular solid mechanics: cells, tissues, and organs. Springer (2002)

48. Huo, Y., Guo, X., Kassab, G.S.: The flow field along the entire length of mouse aorta and primary branches. Annals of Biomedical Engineering 36(5), 685-699 (2008)

49. Ismail, M., Gravemeier, V., Comerford, A., Wall, W.: A stable approach for coupling multidimensional cardiovascular and pulmonary networks based on a novel pressure-flow rate or pressure-only neumann boundary condition formulation. International Journal for Numerical Methods in Biomedical Engineering 30(4), 447-469 (2014)

50. Ismail, M., Wall, W.A., Gee, M.W.: Adjoint-based inverse analysis of windkessel parameters for patientspecific vascular models. Journal of Computational Physics 244, 113-130 (2013)

51. Johnston, B.M., Johnston, P.R., Corney, S., Kilpatrick, D.: Non-newtonian blood flow in human right coronary arteries: steady state simulations. Journal of Biomechanics 37(5), 709-720 (2004)

52. Južnič, G., Klensch, H.: Vergleichend-physiologische Untersuchungen über das Verhalten der Indices für Energieaufwand und Leistung des Herzens. Pflüger's Archiv für die gesamte Physiologie des Menschen und der Tiere 280(1), 38-45 (1964)

53. Karimi, R., Zhu, T., Bouma, B.E., Mofrad, M.R.K.: Estimation of nonlinear mechanical properties of vascular tissues via elastography. Cardiovascular Engineering 8(4), 191-202 (2008) 
54. Karner, G., Perktold, K.: Effect of endothelial injury and increased blood pressure on albumin accumulation in the arterial wall: a numerical study. Journal of Biomechanics 33(6), 709-715 (2000)

55. Kedem, O., Katchalsky, A.: Thermodynamic analysis of the permeability of biological membranes to nonelectrolytes. Biochimica et Biophysica Acta 27, 229-246 (1958)

56. Klisch, S.M., Van Dyke, T.J., Hoger, A.: A theory of volumetric growth for compressible elastic biological materials. Mathematics and Mechanics of Solids 6(6), 551-575 (2001)

57. Klöppel, T., Popp, A., Küttler, U., Wall, W.A.: Fluidstructure interaction for non-conforming interfaces based on a dual mortar formulation. Computer Methods in Applied Mechanics and Engineering 200(45), 3111-3126 (2011)

58. Koshiba, N., Ando, J., Chen, X., Hisada, T.: Multiphysics simulation of blood flow and ldl transport in a porohyperelastic arterial wall model. Journal of Biomechanical Engineering 129(3), 374-385 (2007)

59. Ku, D.N., Giddens, D.P., Zarins, C.K., Glagov, S.: Pulsatile flow and atherosclerosis in the human carotid bifurcation. positive correlation between plaque location and low oscillating shear stress. Arteriosclerosis, Thrombosis, and Vascular Biology 5(3), 293-302 (1985)

60. Kuhl, E., Maas, R., Himpel, G., Menzel, A.: Computational modeling of arterial wall growth. Biomechanics and Modeling in Mechanobiology 6(5), 321-331 (2007)

61. Küttler, U., Gee, M., Förster, C., Comerford, A., Wall, W.: Coupling strategies for biomedical fluid-structure interaction problems. International Journal for Numerical Methods in Biomedical Engineering 26(3-4), 305-321 (2010)

62. Kuzmin, D.: A guide to numerical methods for transport equations. University Erlangen-Nuremberg (2010)

63. Lee, S.W., Antiga, L., Spence, J.D., Steinman, D.A.: Geometry of the carotid bifurcation predicts its exposure to disturbed flow. Stroke 39(8), 2341-2347 (2008)

64. Liu, M., Tang, H., Nicholson, J.K., Lindon, J.C.: Use of $1 \mathrm{~h}$ nmr-determined diffusion coefficients to characterize lipoprotein fractions in human blood plasma. Magnetic Resonance in Chemistry 40(13), S83-S88 (2002)

65. Liu, X., Fan, Y., Deng, X.: Effect of spiral flow on the transport of oxygen in the aorta: a numerical study. Annals of Biomedical Engineering 38(3), 917-926 (2010)

66. Liu, X., Fan, Y., Deng, X., Zhan, F.: Effect of nonnewtonian and pulsatile blood flow on mass transport in the human aorta. Journal of Biomechanics 44(6), 1123-1131 (2011)

67. Liu, Y., Dang, C., Garcia, M., Gregersen, H., Kassab, G.S.: Surrounding tissues affect the passive mechanics of the vessel wall: theory and experiment. American Journal of Physiology-Heart and Circulatory Physiology 293(6), H3290-H3300 (2007)

68. Loree, H.M., Tobias, B.J., Gibson, L.J., Kamm, R.D., Small, D.M., Lee, R.T.: Mechanical properties of model atherosclerotic lesion lipid pools. Arteriosclerosis, Thrombosis, and Vascular Biology 14(2), 230-234 (1994)

69. Lutgens, E., Lievens, D., Beckers, L., Wijnands, E., Soehnlein, O., Zernecke, A., Seijkens, T., Engel, D., Cleutjens, J., Keller, A.M., et al.: Deficient cd40-traf6 signaling in leukocytes prevents atherosclerosis by skewing the immune response toward an antiinflammatory profile. The Journal of Experimental Medicine 207(2), 391-404 (2010)
70. Mayr, M., Klöppel, T., Wall, W.A., Gee, M.W.: A temporal consistent monolithic approach to fluid-structure interaction enabling single field predictors. SIAM Journal on Scientific Computing 37(1), B30-B59 (2015)

71. Moireau, P., Xiao, N., Astorino, M., Figueroa, C.A., Chapelle, D., Taylor, C.A., Gerbeau, J.F.: External tissue support and fluid-structure simulation in blood flows. Biomechanics and Modeling in Mechanobiology 11(1-2), 1-18 (2012)

72. Nadkarni, S.K., Bouma, B.E., Helg, T., Chan, R., Halpern, E., Chau, A., Minsky, M.S., Motz, J.T., Houser, S.L., Tearney, G.J.: Characterization of atherosclerotic plaques by laser speckle imaging. Circulation 112(6), 885-892 (2005)

73. Ogden, R.: Large deformation isotropic elasticity-on the correlation of theory and experiment for incompressible rubberlike solids. In: Proceedings of the Royal Society of London A: Mathematical, Physical and Engineering Sciences, vol. 326, pp. 565-584. The Royal Society (1972)

74. Ogden, R.: Nearly isochoric elastic deformations: application to rubberlike solids. Journal of the Mechanics and Physics of Solids 26(1), 37-57 (1978)

75. Olgac, U., Poulikakos, D., Saur, S.C., Alkadhi, H., Kurtcuoglu, V.: Patient-specific three-dimensional simulation of ldl accumulation in a human left coronary artery in its healthy and atherosclerotic states. American Journal of Physiology-Heart and Circulatory Physiology 296(6), H1969-H1982 (2009)

76. Olshanskii, M., Lube, G., Heister, T., Löwe, J.: Grad-div stabilization and subgrid pressure models for the incompressible navier-stokes equations. Computer Methods in Applied Mechanics and Engineering 198(49), 3975-3988 (2009)

77. Olufsen, M.S., Peskin, C.S., Kim, W.Y., Pedersen, E.M., Nadim, A., Larsen, J.: Numerical simulation and experimental validation of blood flow in arteries with structured-tree outflow conditions. Annals of Biomedical Engineering 28(11), 1281-1299 (2000)

78. Ougrinovskaia, A., Thompson, R.S., Myerscough, M.R.: An ode model of early stages of atherosclerosis: mechanisms of the inflammatory response. Bulletin of Mathematical Biology 72(6), 1534-61 (2010). DOI 10.1007/s11538-010-9509-4

79. Parton, A., McGilligan, V., O'Kane, M., Baldrick, F.R., Watterson, S.: Computational modelling of atherosclerosis. Briefings in Bioinformatics p. bbv081 (2015)

80. Peiffer, V., Sherwin, S.J., Weinberg, P.D.: Does low and oscillatory wall shear stress correlate spatially with early atherosclerosis? a systematic review. Cardiovascular Research p. cvt044 (2013)

81. Prosi, M., Zunino, P., Perktold, K., Quarteroni, A.: Mathematical and numerical models for transfer of low-density lipoproteins through the arterial walls: a new methodology for the model set up with applications to the study of disturbed lumenal flow. Journal of Biomechanics 38(4), 903-917 (2005)

82. Resnick, N., Yahav, H., Shay-Salit, A., Shushy, M., Schubert, S., Zilberman, L.C.M., Wofovitz, E.: Fluid shear stress and the vascular endothelium: for better and for worse. Progress in Biophysics and Molecular Biology 81(3), 177-199 (2003)

83. Roccabianca, S., Figueroa, C., Tellides, G., Humphrey, J.: Quantification of regional differences in aortic stiffness in the aging human. Journal of the Mechanical Behavior of Biomedical Materials 29, 618-634 (2014)

84. Ross, R.: Atherosclerosis - an inflammatory disease. New England Journal of Medicine 340(2), 115-126 (1999) 
85. Saad, Y., Schultz, M.H.: Gmres: A generalized minimal residual algorithm for solving nonsymmetric linear systems. SIAM Journal on Scientific and Statistical Computing 7(3), 856-869 (1986)

86. Sansour, C.: On the physical assumptions underlying the volumetric-isochoric split and the case of anisotropy. European Journal of Mechanics-A/Solids 27(1), 28-39 (2008)

87. Skalak, R., Zargaryan, S., Jain, R.K., Netti, P.A., Hoger, A.: Compatibility and the genesis of residual stress by volumetric growth. Journal of Mathematical Biology 34(8), 889-914 (1996)

88. Smith, N., Pullan, A., Hunter, P.J.: An anatomically based model of transient coronary blood flow in the heart. SIAM Journal on Applied mathematics 62(3), 990-1018 (2002)

89. Soulis, J.V., Lampri, O.P., Fytanidis, D.K., Giannoglou, G.D.: Relative residence time and oscillatory shear index of non-newtonian flow models in aorta. In: Biomedical Engineering, 2011 10th International Workshop on, pp. 1-4. IEEE (2011)

90. de Souza Neto, E., Perić, D., Dutko, M., Owen, D.: Design of simple low order finite elements for large strain analysis of nearly incompressible solids. International Journal of Solids and Structures 33(20), 3277-3296 (1996)

91. Stangeby, D.K., Ethier, C.R.: Computational analysis of coupled blood-wall arterial ldl transport. Journal of Biomechanical Engineering 124(1), 1-8 (2002)

92. Stocker, R., Keaney, J.F.: Role of oxidative modifications in atherosclerosis. Physiological Reviews 84(4), 13811478 (2004)

93. Sun, N., Wood, N.B., Hughes, A.D., Thom, S.A.M., Xu, $X . Y .:$ Influence of pulsatile flow on ldl transport in the arterial wall. Annals of Biomedical Engineering 35(10), 1782-90 (2007). DOI 10.1007/s10439-007-9347-1

94. Suo, J., Ferrara, D.E., Sorescu, D., Guldberg, R.E., Taylor, W.R., Giddens, D.P.: Hemodynamic shear stresses in mouse aortas implications for atherogenesis. Arteriosclerosis, Thrombosis, and Vascular Biology 27(2), 346-351 (2007)

95. Thomas, S.R., Mikulecky, D.C.: Transcapillary solute exchange: A comparison of the kedem-katchalsky convection-diffusion equations with the rigorous nonlinear equations for this special case. Microvascular Research 15(2), 207-220 (1978)

96. Tomaso, G.D., Díaz-Zuccarini, V., Pichardo-Almarza, C.: A multiscale model of atherosclerotic plaque formation at its early stage. IEEE Transactions on Biomedical Engineering 58(12), 3460-3463 (2011)

97. Tompkins, R.G.: Quantitative analysis of blood vessel permeability of squirrel monkeys. American Journal of Physiology - Heart and Circulatory Physiology 260(4), H1194-H1204 (1991)

98. Truskey, G.A., Roberts, W.L., Herrmann, R.A., Malinauskas, R.A.: Measurement of endothelial permeability to $125 \mathrm{i}$-low density lipoproteins in rabbit arteries by use of en face preparations. Circulation Research 71(4), 883-897 (1992)

99. Wada, S., Koujiya, M., Karino, T.: Theoretical study of the effect of local flow disturbances on the concentration of low-density lipoproteins at the luminal surface of end-to-end anastomosed vessels. Medical and Biological Engineering and Computing 40(5), 576-587 (2002)

100. Wall, W., Gee, M.: Baci: a parallel multiphysics finite element environment. Institute for Computational Mechanics, Technische Universität München (2010)
101. Wang, W., Lee, Y., Lee, C.H.: Review: The physiological and computational approaches for atherosclerosis treatment. International Journal of Cardiology 167(5), 1664-1676 (2013)

102. Westerhof, N., Lankhaar, J.W., Westerhof, B.E.: The arterial windkessel. Medical \& Biological Engineering \& Computing 47(2), 131-141 (2009)

103. Whitesall, S.E., Hoff, J.B., Vollmer, A.P., D'Alecy, L.G.: Comparison of simultaneous measurement of mouse systolic arterial blood pressure by radiotelemetry and tailcuff methods. American Journal of Physiology-Heart and Circulatory Physiology 286(6), H2408-H2415 (2004)

104. Wiesmann, F., Szimtenings, M., Frydrychowicz, A., Illinger, R., Hunecke, A., Rommel, E., Neubauer, S., Haase, A.: High-resolution mri with cardiac and respiratory gating allows for accurate in vivo atherosclerotic plaque visualization in the murine aortic arch. Magnetic Resonance in Medicine 50(1), 69-74 (2003)

105. Xiao, N., Alastruey, J., Alberto Figueroa, C.: A systematic comparison between 1-d and 3-d hemodynamics in compliant arterial models. International Journal for Numerical Methods in Biomedical Engineering 30(2), 204-31 (2014). DOI 10.1002/cnm.2598

106. Yang, N., Vafai, K.: Modeling of low-density lipoprotein (ldl) transport in the artery-effects of hypertension. International Journal of Heat and Mass Transfer 49(5), 850-867 (2006)

107. Yang, Y., Richter, T., Jäger, W., Neuss-Radu, M.: An ale approach to mechano-chemical processes in fluidstructure interactions. International Journal for Numerical Methods in Fluids (2016)

108. Yoshihara, L., Coroneo, M., Comerford, A., Bauer, G., Klöppel, T., Wall, W.: A combined fluid-structure interaction and multi-field scalar transport model for simulating mass transport in biomechanics. International Journal for Numerical Methods in Engineering 100(4), 277-299 (2014)

109. Zareh, M., Fradet, G., Naser, G., Mohammadi, H.: Are two-dimensional images sufficient to assess the atherosclerotic plaque vulnerability: a viscoelastic and anisotropic finite element model. Cardiovascular System 3(1), 3 (2015)

110. Zunino, P.: Mathematical and numerical modeling of mass transfer in the vascular system. Ph.D. thesis, Politecnico di Milano (2002) 